\title{
ETHNO-BOTANICAL EVALUATION OF INDIGENOUS FLORA FROM THE COMMUNITIES OF RAJH MEHAL AND GOI UNION COUNCILS OF DISTRICT KOTLI, AZAD JAMMU KASHMIR PAKISTAN
}

\author{
QASEeM, M. F. ${ }^{1}$ - QURESHI, R. ${ }^{1}$ - AMJAD, M. S. ${ }^{2}$ - AhMED, W. ${ }^{1}$ - MASOOD, A. ${ }^{3}$ - ShaHEEN, H. ${ }^{4 *}$ \\ ${ }^{I}$ Department of Botany, Pir Mehr Ali Shah Arid Agriculture University, Rawalpindi, Pakistan \\ ${ }^{2}$ Department of Botany, Women University of Azad Jammu and Kashmir, Bagh, Pakistan \\ ${ }^{3}$ Department of Biology, Pir Mehr Ali Shah Arid Agriculture University, Rawalpindi, Pakistan \\ ${ }^{4}$ Department of Biosciences, COMSATS Institute of Information Technology \\ Park Road, Chak Shahzad, Islamabad, Pakistan \\ *Corresponding author \\ e-mail: humaira.shaheen@comsats.edu.pk
}

(Received 27 $7^{\text {th }}$ Sep 2018; accepted $14^{\text {th }}$ Nov 2018)

\begin{abstract}
The present study reports ethnobotanical information about plants, which were used by indigenous communities of two union councils of district Kotli AJK. The present study is first report of quantitative ethnobotany from study area. Rajh Mehal and Goi are representative union councils of district Kotli, Azad Jammu and Kashmir having large ranges diversity in habitat and climatic conditions. This district is naturally blessed with highly medicinal plants but unfortunately ethno-botanical information is sporadically known. The study area was surveyed for two consecutive years during moon soon period and ethnobotanical data was collected from 112 local inhabitants using a semi-structured, open ended questionnaire and by free listing method. Several quantitative indices including Informant Consensus Factor, Use Value, Frequency of Citation, Relative Frequency of Citation and Relative Importance Index were used to determine relative usage, benefits and coverage of ethno-medicine. The agreement of homogeneity between the present and previous studies and among the indigenous communities was evaluated using the Jaccard Index. The summary statistics and correlation between use value and relative frequency of citation was calculated in SPSS v 16. The data from free listing method was analyzed by using ANTHROPAC package in $\mathrm{R}$ software. Comparison of indigenous knowledge among different genders and age groups was made using Spearman correlation test, the result showed that men had higher information (14.05) about plant uses than females (8.55) in the study area. Male informants reported $63.8 \%( \pm 10.18)$ of total recorded species and $76 \%( \pm 4.13)$ uses while female informants reported $38.8 \%( \pm 6.06)$ species and $68 \%( \pm 3.65)$ uses. Smith's Salience analysis indicates that most of the plants in the study area were useful against abdominal pain and worms, constipation and pain. Diseases were classified into twelve different disease categories based on International Classification of Diseases (ICD) classification, with Skin and related symptoms (S00-T98) had highest value (0.91) of Informant's Consensus Factor (IC) followed by Circulatory problems (I00-I99) and Diabetes (E10-E14) each having IC value of 0.90 . Aerial parts of 21 plant species were utilized in herbal preparations followed by leaves (20 spp.) and oral mode of admiration was most common among inhabitants. Among all the studied species Zanthoxylum alatum was found highly important with a relative importance value of 93.75 followed by Adhatoda zeylanica with an importance value of 91.67. High dependency of local inhabitants on traditional medicines was confirmed by higher values of informant consensus factor, which showed that people in study area still use herbal medicines as a primary source of their health care. The present study indicated that area was rich in medicinal plant knowledge and there is a need to exploit this information for drug development and pharmacological activities in addition of conservation and management of this valuable plant resource of the area.
\end{abstract}

Keywords: ANTHROPAC, frequency of citation, relative importance, informants consensus factor, medicinal importance 
Abbreviations: prior informed consent (PIC), informant consensus (IC), use value index (UV), relative frequency of citation (RFC), medicinal importance (MI), number of use-reports (Nur), number of species cited $(\mathrm{Nt})$, frequency of citation (FC), international plant name index (IPNI), international classification of diseases (ICD), relative pharmacological properties (PH), relative body system treated (BS), relative importance (RI), oral (Or), tropical (Tr)

\section{Background}

One of survey by World Health Organization estimates that about 80 percent of world population depends upon herbal medicines made from approximately 50,000 flowering plants of total world flora (Govaerts, 2001; Schipmann et al., 2002). Effectiveness of plants to cure various aliments is known to humans right from their origin and many herbal drugs are still being utilized in current day herbal system effective against various diseases (Goyal et al., 2014). Indigenous knowledge about plants is an important asset that is gathered from countless activities involving empirical observation, 'trial and error' by the indigenous peoples seeking a cure for an ailment (Locher, 2013). Traditionally, ethnobotanists are interested in exploring the effect of different demographic factors, i.e. age, gender, ethnicity and other attributes of indigenous knowledge coupled with quantitative ethnomedicinal documentation of traditional knowledge by employing different statistical tools (Ullah et al., 2013).

Ethnobotanical knowledge is considered to be an integral part of the knowledge for the drug development. It either helps in identifying new drugs or refocusing on the plants earlier reported to have bioactive components (Mahwasane et al., 2013). The indigenous communities residing in Pakistan mostly use herbal therapies to cure their ailments. Resultantly various studies significantly contributed ethno-pharmacological inventories. With reference to Azad Jammu and Kashmir, some ethnomedical studies have been reported from various areas such as Neelum (Mahmood et al., 2011), Poonch valley (Khan et al., 2010, 2012) and Sudhnoti (Ishtiaq et al., 2015). Few papers have been written on ethnobotanical aspects form the District Kotli (Ajaib et al., 2010; Amjad et al., 2015; Amjad and Arshad, 2014); however, these studies are superficial and lack of novelty in terms of quantitative data in addition to traditional phytotherapies. Therefore, the present study was aimed to:

1. Record and discuss knowledge on medicinal plant uses in the local traditional practices including method of preparation and use of plants as herbal medicine

2. The differential distribution of knowledge about plants and medical properties among sectors of the society

3. Identifying novel uses of plants compared to previously reported work

4. Analysis of ethnobotanical data by using different quantitative indices

\section{Methods}

The study was organized into three stages (Fig. 1).

\section{a) Field work}

The study area lies near line of control and is diversity hotspot due to frequent rain and pleasant weather conditions. Being remote and having less literacy rate people in area still depend upon herbal medicines but documentation of this traditional knowledge is very rare. The present study was conducted by interviewing 112 local inhabitants 
with age ranges between 40 to 75 years, including herbalists, midwives (Daai) and local peoples (male, female). Legal permission was taken from representatives of the municipality for conducting interviews and collection of ethnobotanical data. All respondents were asked to sign a prior informed- consent (PIC) form after the objectives and possible consequences of the study had been explained. The prior informed consent (PIC) form was translated into the local Pahari language. The study area was explored for three years (July 2015-September 2016), plant samples were collected and information was gathered by group discussions, interviews and field visits. Sample size was determined by using method developed by Kadam and Bhalerao (2010).

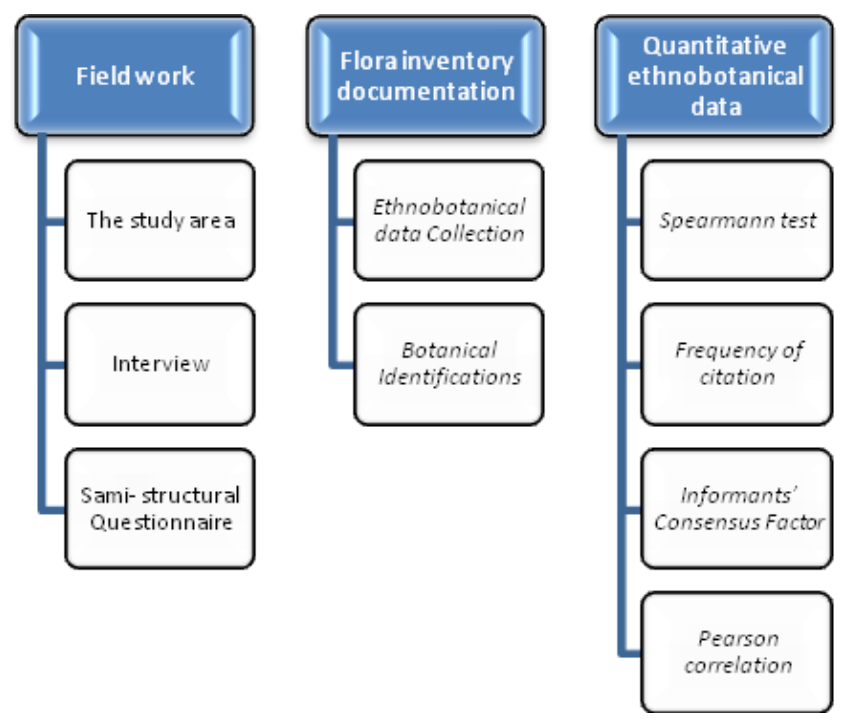

Figure 1. Experimental design for the collection of data

\section{b) Floral inventory documentation}

Ethnobotanical information collected through interviews was recorded in a semistructured questionnaire. Information obtained was cross-checked and compared with existing available literature. Furthermore, the indigenous learning about the plant assets, religious and social perspective, for example, populace density was additionally recorded. The Minimum Standards of Reporting Checklist contains details of the experimental design, and statistics, and resources used in this study. During field surveys, the local/common names of plants were recorded with the help of a local guide or local informants. For taxonomic confirmation of plant was done using different Floras and monographs of the different areas of Pakistan, whereas the International Plant Name Index (IPNI) (www.ipni.org) was also used to obtain the correct botanical name confirmation.

\section{c) Quantitative analyses of ethnobotanical data}

\section{Frequency of citation $(F C)$}

The result of FC presented as \%age and proportions. Each plant reported by informants with respect to the native use was calculated as frequency of citation (FC). The collected ethnomedicinal information was quantitatively analyzed using relative frequency citation index (RFC) after Vijaykumar et al. (2015) as follows: 


$$
\mathrm{RFC}=\mathrm{FC} / \mathrm{N}
$$

where $\mathrm{FC}$ is frequency of citation $\mathrm{N}$ is number of informants.

Informants' consensus factor $\left(F_{I C}\right)$

IC is utilized to build up the consistency of learning among the sources for each sickness treated and diverse illness categories (Heinrich et al., 1998). Informant consensus factor was drawn to search agreements of the informants against the cures for each diseases category. For this purpose, following formula was used:

$$
\mathrm{F}_{\mathrm{IC}}=\frac{N u r-N t}{N u r-1}
$$

where Nur is the number of use-reports in each group of disease; $N t$ is the number of species cited for the particular disease by all the informants. The FIC values are ranged between 0 and 1, in which ' 1 ' shows the maximum level of consensus. Consequently, high $F_{I C}$ can be used in identifying important plants for searching novel bioactive molecules (Carrió and Vallès, 2012).

Medicinal importance (MI)

The MI was figured out by utilizing following equation after Baydoun et al. (2015).

$$
\mathrm{MI}=\frac{\text { No. of use reports for disease category }}{\text { No. of species used }}
$$

Use value index $(U V)$

The use value was calculated by using following formula (Vijayakumar et al., 2015):

$$
\mathrm{UV}=\sum \mathrm{Ui} / \mathrm{N}
$$

$\mathrm{Ui}$ is the number of uses stated by each informant for a given species and $\mathrm{N}$ is the total number of sources (informants).

Relative importance $(R I)$

RI was determined by the formula

$$
\begin{gathered}
\mathrm{RI}=\frac{\mathrm{Rel} \mathrm{PH}+\mathrm{Rel} \mathrm{BS} \times 100}{2} \\
\text { Rel PH }=\frac{\mathrm{PH} \text { of a given plant }}{\text { Maximum PH of all reported plant species }}
\end{gathered}
$$

where $\mathrm{PH}$ is pharmacological attributes of the each provided plant and $\mathrm{Rel} \mathrm{PH}$ is the relative number of pharmacological properties for an individual plant. 


$$
\text { BS }=\frac{\text { BS of a given Plant }}{\text { Maxi. BS of all reported plant species } \$}
$$

BS is the number of body system healed by single species and Rel $\mathrm{PH}$ is relative number of body system treated by a single species.

\section{Smith's salience analysis (SS)}

The analysis of agreement and variation in free-lists using cultural domain analysis is described and justified in detail by Puri (2010), Quinlan (2005), Weller and Baer (2002), and several statistical software packages, including ANTHROPAC, can manage and analyze data in this way. The cultural domain analysis using ANTHROPAC was performed using Borgatti's (1996) Methods Manual. The main steps can be briefly summarized free-lists are compiled in an informant by disease matrix, which indicate both the rank order and the presence or absence of items in each informant's list from this, the frequency of items, their average rank in the list, and a measure of salience (based on Smith, 1993) can be calculated. This is essentially a weighted average of the (inverse) rank of an item across multiple free-lists, where the number of items in the list weights each list.

\section{Pearson correlation}

Pearson correlation analysis was calculated in order to access the linear relationship $\mathrm{b} / \mathrm{w}$ the RFC and Use value index (UV) by using SPSS ver. (16, the $\mathrm{r}^{2}$ ) also estimated to measure cross species variability in RFC that was described by variance in UV. The $\mathrm{r}^{2}$ was computed simply taking square of r (Amjad et al., 2017).

\section{Spearman test}

To decide if a statistically significant correlation exists between the number of plants mentioned and the age of informant, we used the Spearman test. The Mann-Whiney U and Kruskal-Wallis tests were used to find significant differences among two and 5 groups related groups, respectively, all values set at 0.5 alpha level of significance (Bruschi et al., 2011; Shaheen et al., 2017a).

\section{Results}

\section{Demographic data}

Ten field visits (to complete regular varieties) were done to accumulate ethnobotanical information related to use of medicinal plants. The aggregate length of the field work was roughly about 3 years i.e. from July 2015 to September 2016 and data was recorded form 112 informants (male 67 and females 45). The informants were divided in to four categories based on their gender, age group, education and profession. Mostly herbalists were present in the age group range between 51-60 while least number of informants had age more than 60 years. Literacy rate of study area was very low as most of informants (about 26.7\%) were uneducated and only 5 informants which is $4.46 \%$ of total informants had higher education. On average male informants had more information about the plant uses than female informants, male informants on average know 14.05 species than female who know 8.55 species. Similarly uses known 
by male and female were different, on average males report 6.12 uses of plant while female reports 5.82 uses of the plant (Table 1). Informants of age between 41 and 50 know most plants than other age groups but information about plant use was provided most by informants with age between 51-60 years and more than 60 years as they on average report 6.25 and 9.91 uses of plants. Among educated informants, those having middle (secondary school) education know most of the plant species (20.38) and people with intermediate education (Higher secondary school) know most uses of plant species (7.30) among different professionals' traditional health practitioners know most of the species (23.08 on average) and also reported multiple uses (on average 9.60) of recorded species (Table 1). Many examinations have demonstrated that age and sex are two vital variables to be considered while assessing the appropriation of information inside a gathering of sources (Torres-Avilez et al., 2016; Albuquerque et al., 2011). In any case, not many investigations have broken down the impact of these factors on the appropriation of ethno-medicinal information in Pakistan and none of them utilized factual examinations for approval of the gathered information (Ahmad et al., 2014). Spearman's correlation analysis showed significant positive differences between the age and the number of both mentioned species ( $\mathrm{rs}=0.24 ; \mathrm{p}<0.05)$ and different uses ( $\mathrm{rs}=0.35 ; \mathrm{p}<0.05)$, indicating that there is a trend of older people being more knowledgeable than younger people.

Table 1. Demographic data of informants and knowledge about TAB. $F=$ female; $M=$ male

\begin{tabular}{|c|c|c|c|c|c|c|}
\hline \multirow{2}{*}{ Informants } & \multicolumn{2}{|c|}{$\#$} & \multicolumn{2}{|c|}{ \# Known species } & \multicolumn{2}{|c|}{ \# Cited different uses } \\
\hline & $\mathbf{F}$ & $\mathbf{M}$ & $\mathbf{F}$ & $\mathbf{M}$ & $\mathbf{F}$ & M \\
\hline Total & 45 & 67 & $8.55 \pm 6.06$ & $14.05 \pm 10.18$ & $5.82 \pm 3.65$ & $6.12 \pm 4.13$ \\
\hline \multicolumn{7}{|l|}{ Age class } \\
\hline $18-30$ & \multicolumn{2}{|c|}{17} & \multicolumn{2}{|c|}{$5.50 \pm 4.68$} & \multicolumn{2}{|c|}{$5.35 \pm 2.64$} \\
\hline $31-40$ & \multicolumn{2}{|c|}{27} & \multicolumn{2}{|c|}{$10.16 \pm 8.55$} & \multicolumn{2}{|c|}{$4.52 \pm 2.41$} \\
\hline $41-50$ & \multicolumn{2}{|c|}{23} & \multicolumn{2}{|c|}{$15.57 \pm 12.21$} & \multicolumn{2}{|c|}{$4.52 \pm 3.38$} \\
\hline $51-60$ & \multicolumn{2}{|c|}{30} & \multicolumn{2}{|c|}{$12.32 \pm 7.94$} & \multicolumn{2}{|c|}{$6.25 \pm 4.91$} \\
\hline$>61$ & \multicolumn{2}{|c|}{15} & \multicolumn{2}{|c|}{$12.91 \pm 5.16$} & \multicolumn{2}{|c|}{$9.91 \pm 3.70$} \\
\hline Education level & \multirow{2}{*}{\multicolumn{2}{|c|}{30}} & \multirow{2}{*}{\multicolumn{2}{|c|}{$732 \pm 5.09$}} & \\
\hline Uneducated & & & & & \multicolumn{2}{|c|}{$4.50 \pm 2.68$} \\
\hline Intermediate & \multicolumn{2}{|c|}{25} & \multicolumn{2}{|c|}{$12.39 \pm 6.84$} & & \\
\hline Primary & \multicolumn{2}{|c|}{25} & \multicolumn{2}{|c|}{$12.65 \pm 9.50$} & \multicolumn{2}{|c|}{$7.13 \pm 4.51$} \\
\hline Graduate & \multicolumn{2}{|c|}{12} & \multicolumn{2}{|c|}{$7.60 \pm 3.69$} & \multicolumn{2}{|c|}{$5.50 \pm 3.78$} \\
\hline Middle & \multicolumn{2}{|c|}{15} & 20.38 & 13.49 & 5.23 & 2.42 \\
\hline Post graduate & 5 & & 12.00 & 10.58 & 6.00 & 2.00 \\
\hline Profession & & & & & & \\
\hline Worker & 6 & & 9.40 & 7.40 & 7.40 & 2.30 \\
\hline Traditional health practitioner & 26 & & 23.08 & \pm 8.86 & 9.60 & 4.63 \\
\hline Retired & 10 & & 10.28 & 2.69 & & 3.15 \\
\hline House wife & 15 & & 6.15 & 3.84 & 3.8 & 2.30 \\
\hline Midwifery & 9 & & $14.8^{\prime}$ & $t 5.54$ & 8.0 & 3.16 \\
\hline Labour & 5 & & 6.00 & 1.82 & 3.5 & 1.91 \\
\hline Teacher & 20 & & 6.29 & 2.86 & 3.7 & 2.05 \\
\hline Agriculturist & 15 & & 6.73 & 3.45 & 4.41 & 2.58 \\
\hline Student & 7 & & 11.58 & \pm 8.96 & 5.99 & 3.90 \\
\hline
\end{tabular}




\section{Ethno floral diversity}

During the ethnobotanical survey, 80 plants were explored, which belonged to 69 genera and 50 families. The detailed inventory of medicinal plants along with related information is presented in Table 2. Family Asteraceae contributed maximum to ethnoflora with 9 species, followed by Lamiacea, Rosaceae and Solanaceae (4 species each) (Fig. 2). The greater diversity of a family from study area was related to high occurrence of its members, familiarity of local people with its species and presence of active ingredients in its members which are being utilized to treat various ailments (Arnold, 2015; Mouterde, 1966, 1978, 1983). These results are in accordance with the different studies carried out in different regions of Pakistan and world (Bibi et al., 2014; Khan et al., 2014; Lulekal et al., 2013).

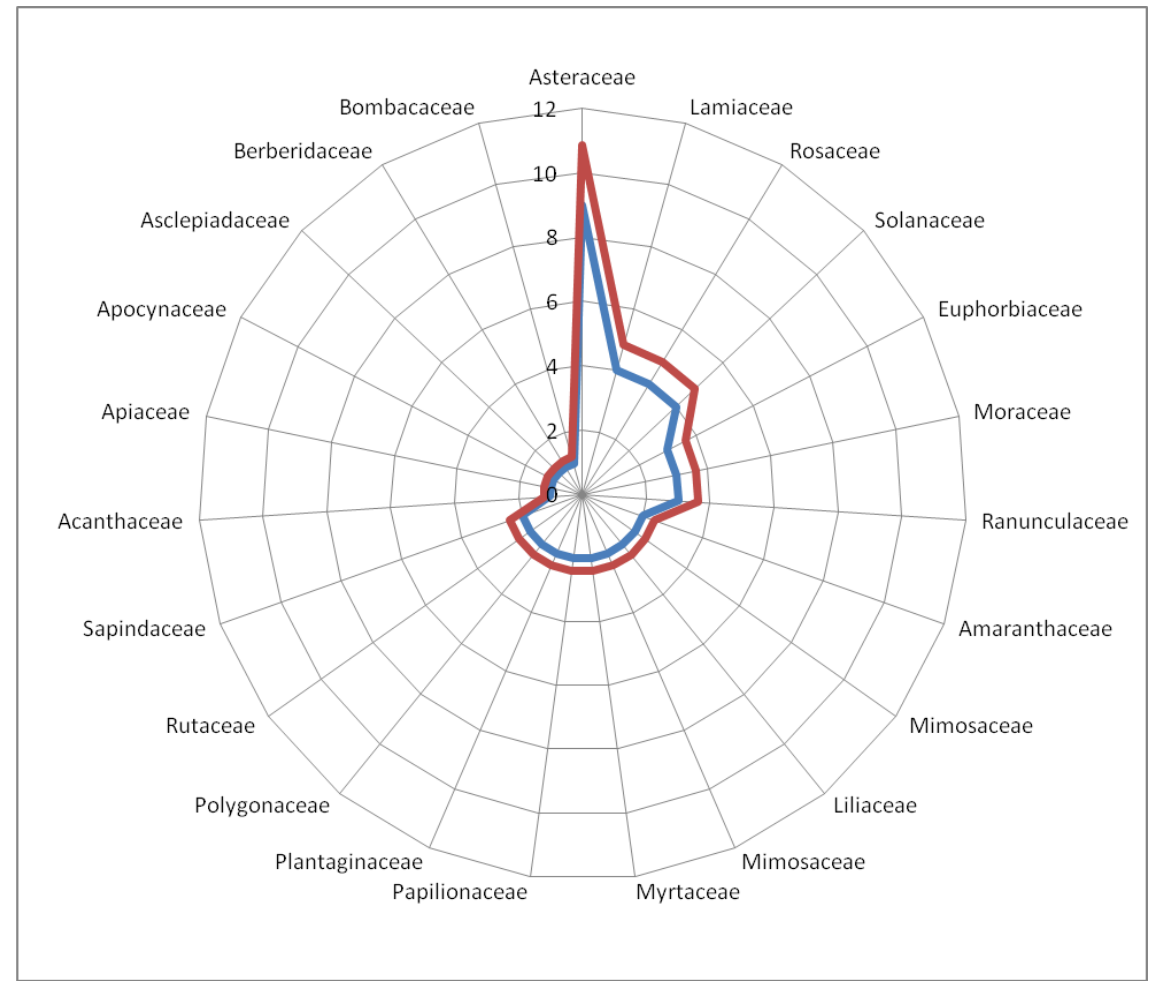

Figure 2. Contribution of major families in the ethnoflora of the study area

\section{Medicinal usage}

In this survey, fifty-three diseases/ailments were treated by 80 plant species with maximum 16 species (30\% of total) were used abdominal pain and constipation which were common and prevailing problems of the area, followed by pain (11 spp., 20.1\%), sexual dysfunction (10 spp., 18.84\%), tooth problems and wounds ( 9 spp., 16.9\% each) (Fig. 3). The documented uses of ethnomedicinal plants were compared with recently published literature (Ishtiaq et al., 2015; Amjad et al., 2017; Shaheen et al., 2017b) to seek the novelty of uses. Most of the documented species possessed similar uses; however, 16 species possessed novel uses (Fig. 4), which were not previously reported in literature. The main reason for the similarity among these studies was the same climatic conditions which resulted into the same floristic composition as well as the dissemination of information related to the use of plants among the informants of 
different areas. The reason for less similarity among the studies may be due to the existence of different cultural values in communities inhabiting the areas so they use plants differently for their healthcare needs. (Baydoun et al., 2015; Panyaphu et al., 2011). However, the informants were unaware of such concern and they informed only beneficial effects of plants without pointing out any side effects. Different parts were used for preparing herbal recipes. Leaves $(31 \%)$ were the most used plant part, followed by aerial parts (17\%), fruit (14\%), roots $(12 \%)$, bark (8\%), flower $(6 \%)$ and bulb, cone branches, pulp, resins (1\% each) (Fig. 5). The preference of leaves in preparing herbal preparations may be due to storage of most of the active ingredients in leaves in order to attain resistance from the herbicides and insects. Furthermore, they are accessible and easy to collect (Panyaphy et al., 2011; Mesfin et al., 2013; Ahmed et al., 2013).

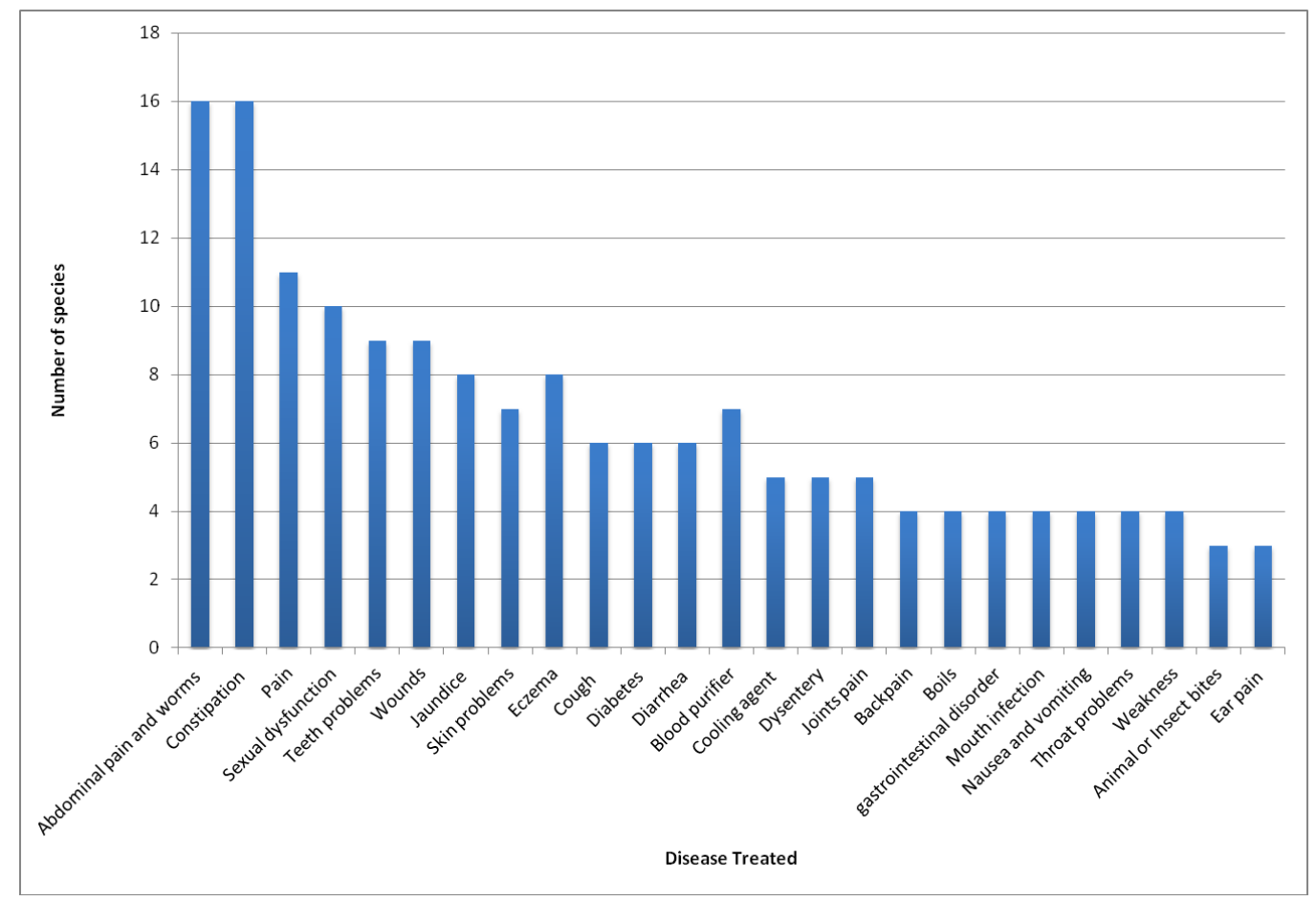

Figure 3. Contribution of ethnoflora for treating different ailments in the study area

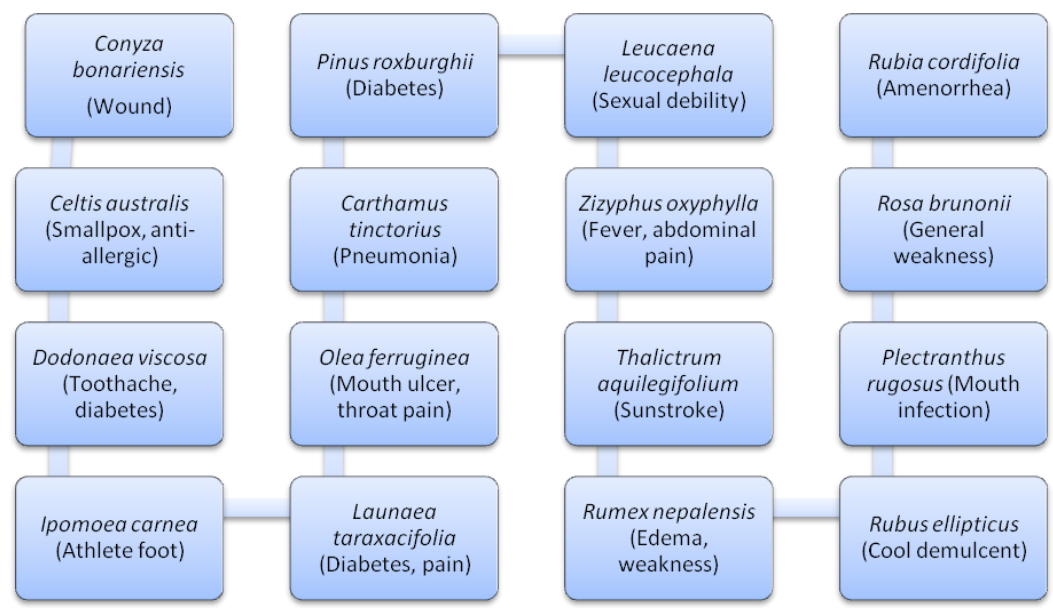

Figure 4. Sixteen plants spp., which have novel uses in the study area 
Table 2. Inventory of medicinal plants including families, plant species, specimen numbers, local names, part used, preparation(s), application, properties/ailment treated, quantitative indices and literature

\begin{tabular}{|c|c|c|c|c|c|c|c|c|c|c|c|c|c|c|c|}
\hline Sr.\# & Family & \begin{tabular}{|c|} 
Plant species/ \\
Specimen number
\end{tabular} & Local name & ${ }^{\text {APart }}$ used & ${ }^{B}$ Preparation & ${ }^{\mathrm{c}}$ Application & ${ }^{D}$ Properties/Aliment treated & ${ }^{\mathrm{E}} \mathbf{F C}$ & ${ }^{\mathrm{F}} \mathrm{RFC}$ & ${ }^{6} \mathbf{U i}$ & ${ }^{\mathrm{H}} \mathbf{U V}$ & IRel. PH & ${ }^{\mathrm{J}}$ Rel. BS & ${ }^{\mathrm{K}_{\mathrm{RI}}}$ & Literature \\
\hline 1 & Acanthaceae & $\begin{array}{l}\text { Adhatoda zeylanica } \\
\text { Medic./KD-24 }\end{array}$ & Baiker & rete & Powder & Or & $\begin{array}{c}\text { Abdominal pain (NI 14), asthma (NI } \\
\text { 12), cough (NI 9), rheumatic pain } \\
\text { (NI 6), ear infection (NI 13), *insect } \\
\text { repellent (NI 5) }\end{array}$ & 59 & 0.79 & 83 & 1.11 & 0.75 & 0.83 & 79.17 & {$[10,45]$} \\
\hline 2 & Amaranthaceae & $\begin{array}{c}\text { Achyranthes aspera } \\
\text { var. } \\
\text { perphyristachya } \\
\text { Hook.f. /KD-47 }\end{array}$ & Puthkanda & करते & Paste, tea & $\operatorname{Tr}$ & $\begin{array}{l}\text { *Pain and inflammation (NI 7), } \\
\text { kidney stone (NI 6), insect bite (NI } \\
\text { 2), bleeding (NI 3), fever (NI 5) }\end{array}$ & 23 & 0.31 & 59 & 0.79 & 0.63 & 0.67 & 64.58 & [46-49] \\
\hline 3 & Amaranthaceae & $\begin{array}{c}\text { Amaranthus viridis } \\
\text { L./ KD-97 }\end{array}$ & Ghanar & & Powder & $\operatorname{Tr}$ & $\begin{array}{l}\text { *Hot tonic (NI: 14), piles (NI: 6), } \\
\text { gonorrhea (NI: 7), Stomach problems } \\
\text { (NI: 9) }\end{array}$ & 36 & 0.48 & 63 & 0.84 & 0.63 & 0.50 & 56.25 & {$[11,49,50]$} \\
\hline 4 & Apiaceae & $\begin{array}{l}\text { Daucus carota } \\
\text { L./KD-58 }\end{array}$ & Gajar & (1) & Juice, extract & Or & $\begin{array}{c}\text { Weak eye sight (NI: 17), stimulants } \\
\text { of the uterus (NI 4), anemia (NI: 10), } \\
\text { blood purifier (NI: 8) }\end{array}$ & 39 & 0.52 & 48 & 0.64 & 0.38 & 0.50 & 43.75 & {$[50,51]$} \\
\hline 5 & Apocynaceae & $\begin{array}{l}\text { Carissa opaca } \\
\text { Stapf. ex } \\
\text { Haines/KD-43 }\end{array}$ & Garanda & & Powder & $\operatorname{Tr}$ & $\begin{array}{l}\text { *Abdominal pain (NI 13), jaundice } \\
\text { (NI 9), sexual debility (NI 4), skin } \\
\text { infection (NI 2), earache (Ni 10) }\end{array}$ & 53 & 0.71 & 78 & 1.04 & 0.75 & 1.00 & 87.50 & {$[52,53]$} \\
\hline 6 & Asclepiadaceae & $\begin{array}{l}\text { Calotropis procera } \\
\text { (Aiton) W.T. Aiton/ } \\
\text { KD-62 }\end{array}$ & $\mathrm{Ak}$ & & Latex & $\operatorname{Tr}$ & $\begin{array}{c}\text { *Dog-bite (NI: 9), back pain (NI: 6), } \\
\text { infection (NI: 9), *sexual problems } \\
\text { (NI: 5), ring worm (NI: 2), asthma } \\
\text { (NI: 7), insect bite ( NI: 4) } \\
\end{array}$ & 42 & 0.56 & 61 & 0.81 & 0.88 & 0.67 & 77.08 & {$[48,49]$} \\
\hline \multirow[t]{2}{*}{7} & \multirow[t]{2}{*}{ Asteraceae } & \multirow[t]{2}{*}{$\begin{array}{c}\text { Artemisia } \\
\text { absinthium L. }\end{array}$} & \multirow[t]{2}{*}{ Babuna } & & $\begin{array}{l}\text { Decoction, } \\
\text { powder }\end{array}$ & Or & $\begin{array}{l}\text { Stomach pain ( NI: 6), earache (NI: } \\
\text { 4), kill worms (NI: 2), inflammation } \\
\text { (NI: 5) }\end{array}$ & \multirow[t]{2}{*}{21} & \multirow[t]{2}{*}{0.28} & \multirow[t]{2}{*}{36} & \multirow[t]{2}{*}{0.48} & \multirow[t]{2}{*}{0.63} & \multirow[t]{2}{*}{0.67} & \multirow[t]{2}{*}{33.33} & \multirow[t]{2}{*}{ [47] } \\
\hline & & & & & & & *Insect repellent (NI: 4) & & & & & & & & \\
\hline
\end{tabular}




\begin{tabular}{|c|c|c|c|c|c|c|c|c|c|c|c|c|c|c|c|}
\hline Sr.\# & Family & $\begin{array}{c}\text { Plant species/ } \\
\text { Specimen number }\end{array}$ & Local name & ${ }^{A}$ Part used & ${ }^{B}$ Preparation & ${ }^{\mathrm{c}}$ Application & ${ }^{D}$ Properties/Aliment treated & ${ }^{\mathrm{E}} \mathrm{FC}$ & ${ }^{\mathrm{F}} \mathrm{RFC}$ & ${ }^{\mathrm{G}} \mathbf{U i}$ & ${ }^{\mathrm{H}} \mathbf{U V}$ & ${ }^{\mathrm{I}} \mathrm{Rel} . \mathbf{P H}$ & ${ }^{\mathrm{J}}$ Rel. BS & ${ }^{\mathrm{K}} \mathbf{R I}$ & Literature \\
\hline 8 & Asteraceae & $\begin{array}{c}\text { Carthamus } \\
\text { tinctorius } \mathrm{L} . / \mathrm{KD}- \\
93 \\
\end{array}$ & $\begin{array}{l}\text { Kasumba } \\
\text { (zafran) }\end{array}$ & & Boiled in oil & $\operatorname{Tr}$ & $\begin{array}{c}\text { *Pneumonia (NI: } 8 \text { ), fever (NI: } 12 \text { ), } \\
\text { cough (NI: 5), throat problems (NI: } \\
6 \text { ) }\end{array}$ & 31 & 0.41 & 52 & 0.69 & 0.50 & 0.50 & 50.00 & [54] \\
\hline 9 & Asteraceae & $\begin{array}{l}\text { Cichorium intybus } \\
\text { L./ KD-61 } \\
\end{array}$ & Kahsni & & Juice & Or & $\begin{array}{c}\text { Fever (NI 5), abdominal disorders } \\
\text { (NI 3), indigestion (NI 4) }\end{array}$ & 12 & 0.16 & 26 & 0.35 & 0.38 & 0.33 & 35.42 & {$[8,47]$} \\
\hline 10 & Asteraceae & \begin{tabular}{|c|} 
Conyza bonariensis \\
(L.) Cronquist/ KD- \\
152 \\
\end{tabular} & & & Powder & $\operatorname{Tr}$ & $\begin{array}{c}\text { Wound (NI 4), bleeding piles (NI 4), } \\
\text { painful menstruation (NI 2) }\end{array}$ & 10 & 0.13 & 15 & 0.20 & 0.38 & 0.50 & 43.75 & [55] \\
\hline 11 & Asteraceae & \begin{tabular}{|c|} 
Launaea \\
taraxacifolia \\
(Willd.) Amin/ KD- \\
89 \\
\end{tabular} & Hand & & Potherb & Or & *Diabetes (NI: 9), pain (NI 6) & 15 & 0.20 & 25 & 0.33 & 0.25 & 0.33 & 29.17 & [56] \\
\hline 12 & Asteraceae & $\begin{array}{l}\text { Sonchus asper }(\mathrm{L} .) \\
\text { Hill./ KD-81 }\end{array}$ & Hundh & & Powder & Or & *Diabetes (NI 7), ${ }^{*}$ body pain (NI 5) & 12 & 0.16 & 18 & 0.24 & 0.25 & 0.33 & 29.17 & [57] \\
\hline 13 & Asteraceae & Tagetes minuta $\mathrm{L}$. & Sadberga & & Extract & Or & $\begin{array}{l}\text { Kidney pain (NI 5), expel kidney } \\
\text { stones (NI 4) } \\
\end{array}$ & 9 & 0.12 & 9 & 0.12 & 0.25 & 0.17 & 20.83 & {$[58,59]$} \\
\hline 14 & Asteraceae & $\begin{array}{l}\text { Taraxacum } \\
\text { officinale F.H. } \\
\text { Wigg/ KD-26 } \\
\end{array}$ & Khali Hand & & Potherb, paste & Or & $\begin{array}{l}\text { *Fever (NI 8), jaundice (NI 3), } \\
\text { swelling (NI 11), joints pain (NI 5) }\end{array}$ & 27 & 0.36 & 64 & 0.85 & 0.50 & 0.50 & 50.00 & [49] \\
\hline 15 & Berberidaceae & $\begin{array}{l}\text { Berberis lycium } \\
\text { DC./ KD-74 }\end{array}$ & Sumbalu & & $\begin{array}{c}\text { Chewed, paste, } \\
\text { extract }\end{array}$ & $\operatorname{Tr}$ & \begin{tabular}{|c|} 
*Mouth inflammation (NI 11), \\
jaundice (NI 9), injury (NI 3), \\
wounds (NI 6), tonic (NI 5), diarrhea \\
(NI 9), rheumatism (NI 7), diabetes \\
(NI 8), \\
\end{tabular} & 58 & 0.77 & 89 & 1.19 & 1.00 & 0.67 & 83.33 & $\begin{array}{c}{[46,48,60]} \\
{[10]}\end{array}$ \\
\hline 16 & Bombacaceae & $\begin{array}{c}\text { Bombax ceiba } \mathrm{L} . / \\
\mathrm{KD}-136 \\
\end{array}$ & Simble & & Powder & Or & $\begin{array}{l}\text { Weakness (NI 11), dysentery (NI 7), } \\
\text { diarrhea (NI13), impotence (NI 8) }\end{array}$ & 39 & 0.52 & 49 & 0.65 & 0.50 & 0.50 & 50.00 & {$[61,62]$} \\
\hline 17 & Boraginaceae & $\begin{array}{l}\text { Trichodesma } \\
\text { indicum }(\mathrm{L} .) \mathrm{R} . \\
\mathrm{Br} . / \mathrm{KD}-125\end{array}$ & Handusi & & $\begin{array}{l}\text { Cooked with } \\
\text { sugar, Infusion }\end{array}$ & Or & $\begin{array}{l}\text { *Back pain (NI 14), *weakness (NI } \\
\text { 9), swellings (NI 3), *urinary } \\
\text { diseases (NI 7), dysentery (NI 8) }\end{array}$ & 41 & 0.55 & 62 & 0.83 & 0.50 & 0.67 & 58.33 & {$[63-65]$} \\
\hline 18 & Brassicaceae & $\begin{array}{l}\text { Lepidium sativum } \\
\quad \mathrm{L} . / \mathrm{KD}-28\end{array}$ & Haleon & & $\begin{array}{c}\text { Seeds are } \\
\text { boiled in milk }\end{array}$ & Or & $\begin{array}{l}\text { Backache (NI 5), pain (NI 2), } \\
\text { abortion (NI 1) *eye cleaner (NI 4) }\end{array}$ & 13 & 0.17 & 22 & 0.29 & 0.50 & 0.50 & 50.00 & [66] \\
\hline 19 & Cactaceae & $\begin{array}{l}\text { Opuntia dillenii } \\
\text { Haw./ KD-15 }\end{array}$ & Thor & & $\begin{array}{c}\text { leaf made into a } \\
\text { pulp /Paste }\end{array}$ & $\operatorname{Tr}$ & $\begin{array}{l}\text { Ophthalmia (NI 8) *joint pain (NI } \\
10 \text { ) }\end{array}$ & 18 & 0.24 & 35 & 0.47 & 0.25 & 0.33 & 29.17 & {$[8]$} \\
\hline 20 & Caesalpiniaceae & $\begin{array}{l}\text { Cassia fistula } \mathrm{L} . / \\
\text { KD-09 }\end{array}$ & Amaltas & & Juice, decoction & Or & $\begin{array}{c}\text { Abdominal pain (NI 12), constipation } \\
\text { (NI 7), joint pain (NI 9) respiratory } \\
\text { problems (NI10), jaundice (NI 6), } \\
\text { piles (NI 5) }\end{array}$ & 49 & 0.65 & 79 & 1.05 & 0.75 & 0.83 & 79.17 & {$[46,67][11]$} \\
\hline
\end{tabular}




\begin{tabular}{|c|c|c|c|c|c|c|c|c|c|c|c|c|c|c|c|}
\hline Sr.\# & Family & $\begin{array}{c}\text { Plant species/ } \\
\text { Specimen number }\end{array}$ & Local name & ${ }^{A}$ Part used & ${ }^{B}$ Preparation & ${ }^{\mathrm{c}}$ Application & ${ }^{D}$ Properties/Aliment treated & ${ }^{\mathrm{E}} \mathrm{FC}$ & ${ }^{\mathrm{F}} \mathrm{RFC}$ & ${ }^{\mathrm{G}} \mathbf{U i}$ & ${ }^{\mathrm{H}} \mathbf{U V}$ & ${ }^{\mathrm{I}} \mathrm{Rel.}$ PH & ${ }^{\mathrm{J}}$ Rel. BS & ${ }^{\mathrm{K}} \mathbf{R I}$ & Literature \\
\hline 21 & Cannabinaceae & $\begin{array}{c}\text { Cannabis sativa L./ } \\
\text { KD-73 }\end{array}$ & Bahng & & $\begin{array}{c}\text { Paste made } \\
\text { with turmeric } \\
\text { and rape seed/ } \\
\text { smoked } \\
\end{array}$ & $\operatorname{Tr}$ & $\begin{array}{l}\text { Swellings NI } 4 \text {, sedative (NI 7), } \\
\text { relief pain (NI 2). }\end{array}$ & 13 & 0.17 & 25 & 0.33 & 0.38 & 0.50 & 43.75 & $\begin{array}{c}{[46,47,68,} \\
69]\end{array}$ \\
\hline 22 & Chenopodiaceae & $\begin{array}{c}\text { Chenopodium } \\
\text { album L./ KD-119 }\end{array}$ & Ghanari & & $\begin{array}{l}\text { Potherb, } \\
\text { decoction, } \\
\text { infusion }\end{array}$ & Or & $\begin{array}{l}\text { Constipation (NI 9), pain in limbs } \\
\text { (NI } 5 \text { ), intestinal problems (NI 12), } \\
\text { jaundice (NI 8), pile (NI 3), throat } \\
\text { and eye problems (NI 9) }\end{array}$ & 46 & 0.61 & 56 & 0.75 & 0.75 & 0.83 & 79.17 & {$[61,47,50]$} \\
\hline 23 & Convolvulaceae & $\begin{array}{l}\text { Ipomoea carnea } \\
\text { Jac./ KD-02 }\end{array}$ & Jungli bakhir & & Paste, decoction & $\operatorname{Tr}$ & $\begin{array}{l}\text { *Athlete foot (NI 5), loose stools } \\
\text { (NI 23) }\end{array}$ & 27 & 0.36 & 24 & 0.32 & 0.25 & 0.33 & 29.17 & {$[50,53]$} \\
\hline \multirow[b]{2}{*}{24} & \multirow[b]{2}{*}{ Cucurbitaceae } & \multirow{2}{*}{$\begin{array}{l}\text { Momordica } \\
\text { charantia } \mathrm{L} . / \mathrm{KD}- \\
22\end{array}$} & \multirow[b]{2}{*}{ Khrella } & & Juice & Or & $\begin{array}{c}\text { Diabetes (NI 6), jaundice (NI 4), } \\
\text { piles (NI 2) }\end{array}$ & \multirow[b]{2}{*}{15} & \multirow[b]{2}{*}{0.20} & \multirow[b]{2}{*}{21} & \multirow[b]{2}{*}{0.28} & \multirow[b]{2}{*}{0.50} & \multirow[b]{2}{*}{0.50} & \multirow[b]{2}{*}{25.00} & \multirow[b]{2}{*}[11,70]{} \\
\hline & & & & & $\begin{array}{c}\text { Leaves coated } \\
\text { with oil } \\
\text { warmed } \\
\end{array}$ & $\operatorname{Tr}$ & Pain/swelling (NI 1) & & & & & & & & \\
\hline \multirow[t]{2}{*}{25} & \multirow[t]{2}{*}{ Cuscutaceae } & \multirow[t]{2}{*}{$\begin{array}{l}\text { Cuscuta reflexa } \\
\text { Roxb./ KD-114 }\end{array}$} & \multirow[t]{2}{*}{ Neeladari } & & $\begin{array}{l}\text { Decoction, } \\
\text { juice }\end{array}$ & Or & $\begin{array}{l}\text { Jaundice (NI 6), blood purifier (NI } \\
\text { 9), Hair fall (NI 11), skin disease } \\
\text { (NI 4) }\end{array}$ & \multirow[t]{2}{*}{38} & \multirow[t]{2}{*}{0.51} & \multirow[t]{2}{*}{62} & \multirow[t]{2}{*}{0.83} & \multirow[t]{2}{*}{0.63} & \multirow[t]{2}{*}{0.67} & \multirow[t]{2}{*}{64.58} & \multirow[t]{2}{*}{ [48] } \\
\hline & & & & & Paste & $\operatorname{Tr}$ & Fractured bones (NI 8) & & & & & & & & \\
\hline \multirow[t]{2}{*}{26} & \multirow[t]{2}{*}{ Euphorbiaceae } & \multirow[t]{2}{*}{$\begin{array}{l}\text { Ricinus communis } \\
\text { L./ KD-10 }\end{array}$} & \multirow[t]{2}{*}{ Arind } & & \begin{tabular}{|c|} 
Leaves coated \\
with mustard \\
oil and warmed/ \\
rapped on \\
abdomen of \\
kids, powder, \\
poultice \\
\end{tabular} & $\operatorname{Tr}$ & $\begin{array}{c}\text { Wounds (NI 7), boils (NI 5), } \\
\text { swellings (NI 3), Joints } \\
\text { pain/inflammation (NI 14), flatulence } \\
\text { (NI 9) Increase milk production in } \\
\text { females (NI 4) }\end{array}$ & \multirow[t]{2}{*}{45} & \multirow[t]{2}{*}{0.60} & \multirow[t]{2}{*}{58} & \multirow[t]{2}{*}{0.77} & \multirow[t]{2}{*}{0.88} & \multirow[t]{2}{*}{0.67} & \multirow[t]{2}{*}{33.33} & {$[48,50]$} \\
\hline & & & & (1.) & $\begin{array}{l}\text { Given with } \\
\text { milk }\end{array}$ & Or & Constipation (NI 3) & & & & & & & & \\
\hline 27 & Euphorbiaceae & \begin{tabular}{|c|} 
Euphorbia \\
helioscopia L./ KD- \\
42 \\
\end{tabular} & Dodak & & Paste & $\operatorname{Tr}$ & $\begin{array}{l}\text { Boils (NI 19), female problems (NI } \\
7 \text { ) }\end{array}$ & 26 & 0.35 & 30 & 0.40 & 0.25 & 0.33 & 29.17 & [13] \\
\hline 28 & Euphorbiaceae & \begin{tabular}{|c|} 
Mallotus \\
philippensis $($ Lam.) \\
Muell.// KD-163 \\
\end{tabular} & Kamilla & & $\begin{array}{c}\text { Powder mixed } \\
\text { with mustard } \\
\text { oil }\end{array}$ & $\operatorname{Tr}$ & $\begin{array}{l}\text { Hair tonic (NI 4), anti-lice (NI 5), } \\
\text { measles mumps (NI 2) }\end{array}$ & 29 & 0.39 & 47 & 0.63 & 0.63 & 0.50 & 25.00 & {$[45,71]$} \\
\hline
\end{tabular}




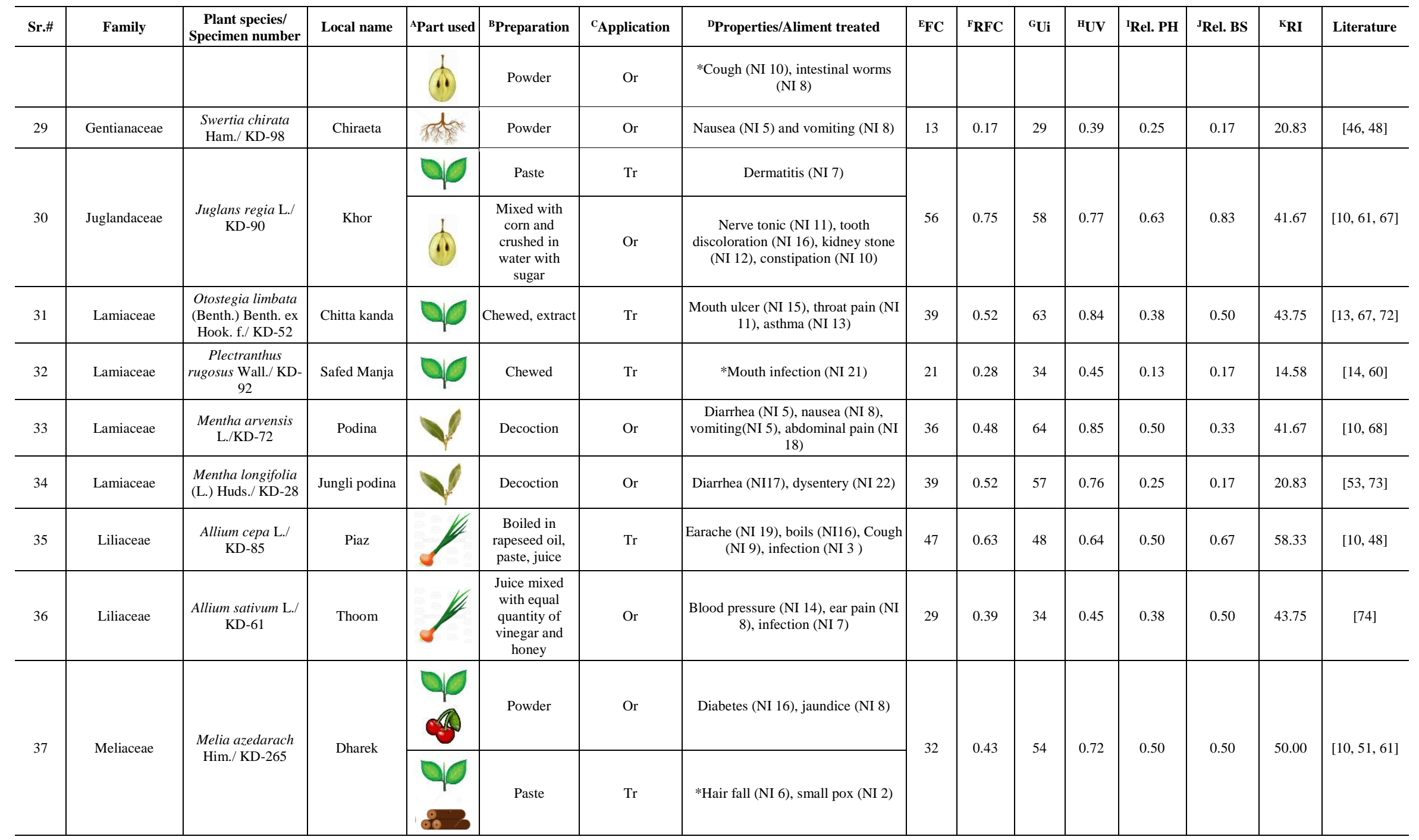




\begin{tabular}{|c|c|c|c|c|c|c|c|c|c|c|c|c|c|c|c|}
\hline Sr.\# & Family & $\begin{array}{c}\text { Plant species/ } \\
\text { Specimen number }\end{array}$ & Local name & ${ }^{A}$ Part used & ${ }^{B}$ Preparation & ${ }^{\mathrm{c}}$ Application & ${ }^{D}$ Properties/Aliment treated & ${ }^{\mathrm{E}} \mathbf{F C}$ & ${ }^{\mathrm{F}} \mathbf{R F C}$ & ${ }^{\mathrm{G}} \mathbf{U i}$ & ${ }^{\mathrm{H}} \mathbf{U V}$ & ${ }^{\text {IRel. PH }}$ & ${ }^{\mathrm{J}}$ Rel. BS & ${ }^{\mathrm{K}_{\mathbf{R I}}}$ & Literature \\
\hline \multirow{2}{*}{38} & \multirow{2}{*}{ Mimosaceae } & \multirow{2}{*}{$\begin{array}{c}\text { Albizia lebbeck }(\mathrm{L} .) \\
\text { Benth./ KD-89 }\end{array}$} & \multirow{2}{*}{ Shrin } & & Paste & Tr, Miswak & $\begin{array}{c}\text { Inflammations (NI 10), burns (NI 9), } \\
\text { toothache (NI 14), strengthens the } \\
\text { gums and teeth (NI 4), }\end{array}$ & & & \multirow{2}{*}{73} & \multirow{2}{*}{0.97} & \multirow{2}{*}{0.63} & \multirow{2}{*}{0.50} & \multirow{2}{*}{56.25} & \multirow{2}{*}{ [75] } \\
\hline & & & & & $\begin{array}{c}\text { Powder mixed } \\
\text { with mint } \\
\text { leaves, } \\
\end{array}$ & Or & *Sexual debility (11), & 48 & 0.64 & & & & & & \\
\hline 39 & Mimosaceae & 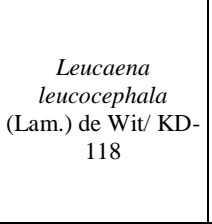 & Kikar & & $\begin{array}{c}\text { Equal quantity } \\
\text { of seeds mixed } \\
\text { with Albizia } \\
\text { lebbeckand } \\
\text { made into } \\
\text { powdered, oil, } \\
\text { decoction }\end{array}$ & Or & $\begin{array}{l}\text { *Sexual debility (NI 14), bacterial } \\
\text { infection (NI 7), contraceptive (NI } \\
\text { 13) }\end{array}$ & 34 & 0.45 & 49 & 0.65 & 0.38 & 0.50 & 43.75 & [76] \\
\hline 40 & Moraceae & $\begin{array}{l}\text { Ficus palmata } \\
\text { Forssk./ KD-168 }\end{array}$ & Phagwara & & $\begin{array}{c}\text { Thin layer } \\
\text { wrapped/ eaten }\end{array}$ & Or & $\begin{array}{l}\text { *Wounds (NI 11), constipation((NI } \\
\text { 9), extraction of spines from flash } \\
\text { (NI 15) }\end{array}$ & 35 & 0.47 & 59 & 0.79 & 0.38 & 0.33 & 35.42 & {$[73,77]$} \\
\hline \multirow[b]{2}{*}{41} & \multirow[b]{2}{*}{ Moraceae } & \multirow[b]{2}{*}{$\begin{array}{l}\text { Ficus benghalensis } \\
\text { L./ KD-260 }\end{array}$} & \multirow[b]{2}{*}{ Bhohar } & & & $\operatorname{Tr}$ & Heel cracks (NI 5) & \multirow[b]{2}{*}{31} & \multirow[b]{2}{*}{0.41} & \multirow[b]{2}{*}{25} & \multirow[b]{2}{*}{0.33} & \multirow[b]{2}{*}{0.50} & \multirow[b]{2}{*}{0.50} & \multirow[b]{2}{*}{50.00} & \multirow[b]{2}{*}{ [11] } \\
\hline & & & & & $\begin{array}{l}\text { Poured into } \\
\text { candy } \\
\text { (Batasha) }\end{array}$ & Or & $\begin{array}{l}\text { *Sexual debility (NI 17), premature } \\
\text { ejaculation (NI 8), Scurvy (NI 1) }\end{array}$ & & & & & & & & \\
\hline 42 & Moraceae & $\begin{array}{l}\text { Morus nigra L./ } \\
\text { KD-81 }\end{array}$ & Kala toot & & $\begin{array}{l}\text { Juice is made } \\
\text { into syrup, } \\
\text { Infusion }\end{array}$ & Or & \begin{tabular}{|c|} 
Cough (NI 13), throat pain (NI 12), \\
inflammation (NI 9), diarrhea (NI 4), \\
Pain (NI 3), diabetes (NI 5),
\end{tabular} & 46 & 0.61 & 64 & 0.85 & 0.75 & 0.83 & 79.17 & {$[10,61][63]$} \\
\hline 43 & Myrtaceae & $\begin{array}{c}\text { Eucalyptus } \\
\text { camaldulensis } \\
\text { Dehnh./KD- } 99 \\
\end{array}$ & & & $\begin{array}{l}\text { Rubbed and } \\
\text { smelled }\end{array}$ & $\operatorname{Tr}$ & $\begin{array}{l}\text { Cold (15), cough(12), }{ }_{(6)} \text { condiment } \\
\text { (6) }\end{array}$ & 33 & 0.44 & 25 & 0.33 & 0.38 & 0.33 & 35.42 & {$[78]$} \\
\hline 44 & Myrtaceae & $\begin{array}{c}\text { Psidium guvajva } \mathrm{L} . / \\
\mathrm{KD}-110\end{array}$ & Amrood & & $\begin{array}{l}\text { Kept in warm } \\
\text { ash for few } \\
\text { minutes, } \\
\text { chewing, } \\
\text { decoction }\end{array}$ & Or & $\begin{array}{l}\text { Flu (NI 9), cough (5), anthelmintic } \\
\text { (14), bleeding gums (NI 5), bad } \\
\text { breath (NI 4), wounds (2), vaginal } \\
\text { irritation and discharges ( } 3 \text { ) }\end{array}$ & 42 & 0.56 & 61 & 0.81 & 0.88 & 0.67 & 77.08 & {$[67,79]$} \\
\hline 45 & Nyctaginaceae & $\begin{array}{c}\text { Boerhavia } \\
\text { procumben } \text { Banks } \\
\text { ex Roxb./ KD-235 }\end{array}$ & Budh bail & & Decoction & Or & $\begin{array}{c}\text { *Refrigerant (NI 6), demulcent drink } \\
\text { (NI 6) }\end{array}$ & 12 & 0.16 & 31 & 0.41 & 0.25 & 0.17 & 20.83 & [47] \\
\hline 46 & Oleaceae & $\begin{array}{l}\text { Olea ferruginea } \\
\text { Royle/KD-21 }\end{array}$ & Kohu & & $\begin{array}{l}\text { Chewed, } \\
\text { toothbrush }\end{array}$ & $\operatorname{Tr}$ & $\begin{array}{l}\text { *Mouth ulcer (NI 17), throat pain (NI } \\
\text { 5), diabetes (NI 4), toothache (NI 8) }\end{array}$ & 53 & 0.71 & 64 & 0.85 & 0.63 & 0.67 & 64.58 & {$[57,80]$} \\
\hline
\end{tabular}

APPLIED ECOLOGY AND ENVIRONMENTAL RESEARCH 17(2):2799-2829.

http://www.aloki.hu • ISSN 15891623 (Print) • ISSN 17850037 (Online)

DOI: http://dx.doi.org/10.15666/aeer/1702_27992829

(c) 2019, ALÖKI Kft., Budapest, Hungary 


\begin{tabular}{|c|c|c|c|c|c|c|c|c|c|c|c|c|c|c|c|}
\hline Sr.\# & Family & \begin{tabular}{|c|} 
Plant species/ \\
Specimen number
\end{tabular} & Local name & ${ }^{A}$ Part used & ${ }^{\mathrm{B}}$ Preparation & ${ }^{\mathrm{c}}$ Application & ${ }^{D}$ Properties/Aliment treated & ${ }^{\mathrm{E}} \mathbf{F C}$ & ${ }^{\mathrm{F}} \mathrm{RFC}$ & ${ }^{\mathrm{G}} \mathrm{Ui}$ & ${ }^{\mathrm{H}} \mathbf{U V}$ & ${ }^{\mathrm{I}} \mathrm{Rel} . \mathbf{P H}$ & ${ }^{J}$ Rel. BS & ${ }^{{ }^{K}} \mathbf{R I}$ & Literature \\
\hline & & & & & Oil & $\operatorname{Tr}$ & Pain (NI 10), inflammation (NI 9) & & & & & & & & \\
\hline \multirow{2}{*}{47} & \multirow{2}{*}{ Oxalidaceae } & \multirow{2}{*}{$\begin{array}{l}\text { Oxalis corniculata } \\
\text { L/ KD-193 }\end{array}$} & Jandouro & & \begin{tabular}{|l} 
Crushed with \\
red chilies \\
$($ Chatni), paste
\end{tabular} & Or & Lack of appetite (NI 15) & \multirow[t]{2}{*}{51} & \multirow{2}{*}{0.68} & \multirow[t]{2}{*}{49} & \multirow{2}{*}{0.65} & \multirow{2}{*}{0.63} & \multirow{2}{*}{0.50} & \multirow{2}{*}{25.00} & \multirow{2}{*}[10,80]{} \\
\hline & & & Jandouro & & Paste, rubbed & $\operatorname{Tr}$ & \begin{tabular}{|} 
*Pain (NI 12), *pus formation (NI 6), \\
Snake bite (NI 8), wounds (NI 10)
\end{tabular} & & & & & & & & \\
\hline 48 & Papilionaceae & $\begin{array}{c}\text { Medicago } \\
\text { polymorpha Willd./ } \\
\text { KD-73 }\end{array}$ & Siree & & Potherb & Or & $\begin{array}{l}\text { Heart tonic (NI 4), constipation (NI } \\
\text { 8), wounds (NI 3) }\end{array}$ & 15 & 0.20 & 25 & 0.33 & 0.38 & 0.50 & 43.75 & {$[49,57]$} \\
\hline \multirow[b]{2}{*}{49} & \multirow[b]{2}{*}{ Papilionaceae } & \multirow[b]{2}{*}{$\begin{array}{l}\text { Dalbergia sissoo } \\
\text { Roxb. / KD-37 }\end{array}$} & \multirow[b]{2}{*}{ Tali } & & Oil, Wrapping & $\operatorname{Tr}$ & $\begin{array}{c}\text { Scabies (NI 3), swelling of the breast } \\
\text { (NI 7) }\end{array}$ & \multirow[b]{2}{*}{35} & \multirow[b]{2}{*}{0.47} & \multirow[b]{2}{*}{34} & \multirow[b]{2}{*}{0.45} & \multirow[b]{2}{*}{0.75} & \multirow[b]{2}{*}{0.83} & \multirow[b]{2}{*}{79.17} & \multirow[b]{2}{*}[67,71]{$[81]$} \\
\hline & & & & & $\begin{array}{l}\text { Decoction, } \\
\text { Juice, powder } \\
\text { mixed with oil }\end{array}$ & Or & $\begin{array}{l}* \text { Cooling agent (NI 10), diarrhea (NI } \\
4 \text { ), dandruff (NI 5), gonorrhea (NI 6), }\end{array}$ & & & & & & & & \\
\hline 50 & Papaveraceae & $\begin{array}{c}\text { Papaver } \\
\text { somniferum } \mathrm{L} . / \mathrm{KD}- \\
187\end{array}$ & Post & & $\begin{array}{l}\text { Roasted fruit } \\
\text { ground with } \\
\text { Terminalia } \\
\text { chebula, } \\
\text { Foeniculum } \\
\text { vulgare and } \\
\text { black salt } \\
\end{array}$ & Or & $\begin{array}{l}\text { Abdominal pain (NI 11), } \\
\text { sleeplessness (NI 6) }\end{array}$ & 17 & 0.23 & 21 & 0.28 & 0.25 & 0.33 & 29.17 & {$[47,68]$} \\
\hline \multirow{2}{*}{51} & \multirow{2}{*}{ Pinaceae } & \multirow{2}{*}{$\begin{array}{l}\text { Pinus roxburghii } \\
\text { Sargent./ KD-203 }\end{array}$} & \multirow{2}{*}{ Chir } & & $\begin{array}{l}\text { Plasters, herbal } \\
\text { steam baths }\end{array}$ & $\operatorname{Tr}$ & $\begin{array}{l}\text { Ringworm (NI } 5 \text { ), boils (NI 7), } \\
\text { rheumatism (NI 6), cough (NI 10), } \\
\text { cold (NI 2) }\end{array}$ & \multirow{2}{*}{45} & \multirow{2}{*}{0.60} & \multirow{2}{*}{56} & \multirow{2}{*}{0.75} & \multirow{2}{*}{0.88} & \multirow{2}{*}{0.67} & \multirow{2}{*}{77.08} & \multirow{2}{*}[10,46,68]{} \\
\hline & & & & & $\begin{array}{c}\text { Soaked } \\
\text { overnight in } \\
\text { water (Juice) }\end{array}$ & Or & $\begin{array}{l}\text { *Diabetes (NI 12), kidney and } \\
\text { bladder problems (NI 3) }\end{array}$ & & & & & & & & \\
\hline 52 & Plantaginaceae & \begin{tabular}{|} 
Plantago major $\mathrm{L} . /$ \\
$\mathrm{KD}-109$
\end{tabular} & Chamchapatar & & $\begin{array}{l}\text { Mixed with } \\
\text { curd, Infusion }\end{array}$ & Or & Dysentery (NI 6), haemoptysis (NI 7) & 13 & 0.17 & 23 & 0.31 & 0.25 & 0.33 & 29.17 & [61] \\
\hline 53 & Plantaginaceae & $\begin{array}{l}\text { Plantago ovata } \\
\text { Forssk./ KD-16 }\end{array}$ & Ismaghol & & Heated leaves & $\operatorname{Tr}$ & Wounds (NI 2), toothache (NI 3) & 10 & 0.13 & 15 & 0.20 & 0.38 & 0.50 & 18.75 & {$[68]$} \\
\hline
\end{tabular}

APPLIED ECOLOGY AND ENVIRONMENTAL RESEARCH 17(2):2799-2829.

http://www.aloki.hu • ISSN 15891623 (Print) • ISSN 17850037 (Online)

DOI: http://dx.doi.org/10.15666/aeer/1702_27992829

( 2019 , ALÖKI Kft., Budapest, Hungary 


\begin{tabular}{|c|c|c|c|c|c|c|c|c|c|c|c|c|c|c|c|}
\hline Sr.\# & Family & \begin{tabular}{|c|} 
Plant species/ \\
Specimen number
\end{tabular} & Local name & ${ }^{A}$ Part used & ${ }^{\text {BPreparation }}$ & ${ }^{\mathrm{c}}$ Application & ${ }^{\mathrm{D}}$ Properties/Aliment treated & ${ }^{\mathrm{E}} \mathbf{F C}$ & ${ }^{\mathrm{F}} \mathrm{RFC}$ & ${ }^{\mathrm{G}} \mathbf{U i}$ & ${ }^{\mathrm{H}} \mathbf{U V}$ & ${ }^{\text {I Rel. PH }}$ & ${ }^{J}$ Rel. BS & ${ }^{\mathrm{K}} \mathbf{R I}$ & Literature \\
\hline & & & & & $\begin{array}{l}\text { Mixed with } \\
\text { curd }\end{array}$ & Or & Dysentery (NI 5) & & & & & & & & \\
\hline \multirow{2}{*}{54} & \multirow{2}{*}{ Poaceae } & \multirow{2}{*}{$\begin{array}{l}\text { Cynodon dactylon } \\
\text { (L.) Pers./ KD-11 }\end{array}$} & \multirow{2}{*}{ Khabal } & & $\begin{array}{l}\text { Infusion along } \\
\text { milk, juice, }\end{array}$ & Or & $\begin{array}{c}\text { Piles (NI 8), bleeding from nose (NI } \\
5 \text { ) }\end{array}$ & \multirow{2}{*}{25} & \multirow{2}{*}{0.33} & \multirow{2}{*}{44} & \multirow{2}{*}{0.59} & \multirow{2}{*}{0.50} & \multirow{2}{*}{0.50} & \multirow{2}{*}{50.00} & \multirow{2}{*}[68,75]{} \\
\hline & & & & & Paste, poultice & $\operatorname{Tr}$ & Cuts (NI 10), wounds (NI 2) & & & & & & & & \\
\hline 55 & Polygonaceae & $\begin{array}{l}\text { Rumex hastatus D. } \\
\text { Don/ KD-121 }\end{array}$ & Ambhi & & Potherb, Juice & Or & $\begin{array}{c}\text { Headache (NI 9), dyspepsia (NI 5), } \\
\text { *flatulence in animals (NI 6), } \\
\text { constipation (NI 9), dysentery with } \\
\text { blood (NI 8) } \\
\end{array}$ & 37 & 0.49 & 33 & 0.44 & 0.63 & 0.33 & 47.92 & {$[61,68,79]$} \\
\hline 56 & Polygonaceae & $\begin{array}{l}\text { Rumex nepalensis } \\
\quad \mathrm{L} . / \mathrm{KD}-22\end{array}$ & Halfari & & \begin{tabular}{|c|} 
Leaves mixed \\
with \\
Nasturtium \\
officinale(Pothe \\
rb), decoction, \\
paste
\end{tabular} & Or & $\begin{array}{c}\text { *Edema (NI 5), weakness (NI 3), } \\
\text { Body pain (NI 2), swollen gums (NI } \\
\text { 2) }\end{array}$ & 12 & 0.16 & 15 & 0.20 & 0.50 & 0.33 & 41.67 & [82] \\
\hline \multirow{3}{*}{57} & \multirow{3}{*}{ Punicaeae } & \multirow{3}{*}{$\begin{array}{l}\text { Punica granatum } \\
\text { var. nana } \mathrm{L} . / \mathrm{KD}-61\end{array}$} & \multirow{3}{*}{ Darunna } & & Powder & Or & * Leucorrhoea (NI 5) & \multirow{3}{*}{55} & \multirow{3}{*}{0.73} & \multirow{3}{*}{58} & \multirow{3}{*}{0.77} & \multirow{3}{*}{1.00} & \multirow{3}{*}{1.00} & \multirow{3}{*}{100} & \multirow{3}{*}{ [13] [83] } \\
\hline & & & & & Powder & Or & Diarrhea (NI 9), dysentery (NI 7) & & & & & & & & \\
\hline & & & & & $\begin{array}{c}\text { Soaked in water } \\
\text { overnight } \\
\text { (Juice) }\end{array}$ & Or & $\begin{array}{l}\text { Jaundice (NI 12), heart disease (NI } \\
\text { 7), blood pressure (NI 6), bleeding } \\
\text { from nose (NI 4), expel worms (NI } \\
\text { 5) }\end{array}$ & & & & & & & & \\
\hline 58 & Ranunculaceae & $\begin{array}{c}\text { Thalictrum } \\
\text { aquilegifolium } \mathrm{L} . / \\
\text { KD-230 }\end{array}$ & Beeni & the & $\begin{array}{l}\text { Crushed in } \\
\text { water (Juice) }\end{array}$ & Or & Sunstroke (NI 9), jaundice (NI 5) & 14 & 0.19 & 25 & 0.33 & 0.25 & 0.33 & 29.17 & {$[84,85]$} \\
\hline \multirow[b]{2}{*}{59} & \multirow[b]{2}{*}{ Ranunculaceae } & \multirow{2}{*}{$\begin{array}{c}\text { Ranunculus } \\
\text { arvensis L./ KD- } \\
198\end{array}$} & \multirow[b]{2}{*}{ Chichumbha } & & Potherb & Or & Indigestion (NI 22) & \multirow[b]{2}{*}{43} & \multirow[b]{2}{*}{0.57} & \multirow[b]{2}{*}{67} & \multirow[b]{2}{*}{0.89} & \multirow[b]{2}{*}{0.38} & \multirow[b]{2}{*}{0.33} & \multirow[b]{2}{*}{18.75} & \\
\hline & & & & & $\begin{array}{c}\text { Boiled in } \\
\text { rapeseed oil and } \\
\text { ground }\end{array}$ & $\operatorname{Tr}$ & *Boils (NI 18), pimples (NI 3) & & & & & & & & {$[10,68,83]$} \\
\hline 60 & Ranunculaceae & $\begin{array}{l}\text { Rananculus } \\
\text { scleratus } \mathrm{L} . / \mathrm{KD}- \\
398\end{array}$ & Jal dhania & & Juice & Or & *Blood purifier (NI 15 ) & 15 & 0.20 & 34 & 0.45 & 0.13 & 0.17 & 14.58 & \\
\hline
\end{tabular}




\begin{tabular}{|c|c|c|c|c|c|c|c|c|c|c|c|c|c|c|c|}
\hline Sr.\# & Family & \begin{tabular}{|c|} 
Plant species/ \\
Specimen number
\end{tabular} & Local name & ${ }^{A}$ Part used & ${ }^{B}$ Preparation & ${ }^{\mathrm{C}}$ Application & ${ }^{D}$ Properties/Aliment treated & ${ }^{\mathrm{E}} \mathbf{F C}$ & ${ }^{\mathrm{F}} \mathrm{RFC}$ & ${ }^{\mathrm{G}} \mathbf{U i}$ & ${ }^{\mathrm{H}} \mathbf{U V}$ & ${ }^{\text {I Rel. PH }}$ & ${ }^{J}$ Rel. BS & ${ }^{\mathrm{K}} \mathbf{R I}$ & Literature \\
\hline 61 & Rhamnaceae & \begin{tabular}{|l} 
Zizyphus oxyphylla \\
Edgew./KD-312
\end{tabular} & Ber & & $\begin{array}{c}\text { Root barks and } \\
\text { Achyranthes } \\
\text { aspera } \\
\text { (Powder) }\end{array}$ & Or & $\begin{array}{l}\text { *Fever (NI 11),* abdominal pain (NI } \\
\text { 5), Diabetes (NI 10), pain (NI 2) }\end{array}$ & 28 & 0.37 & 32 & 0.43 & 0.50 & 0.67 & 58.33 & [86] \\
\hline \multirow{2}{*}{62} & \multirow{2}{*}{ Rosaceae } & \multirow{2}{*}{$\begin{array}{c}\text { Prunus persica }(\mathrm{L} .) \\
\text { Batsch./ KD-291 }\end{array}$} & \multirow{2}{*}{ Arwaari } & & $\begin{array}{l}\text { Cooked with } \\
\text { rice }\end{array}$ & Or & Constipation (NI 13), edema (NI 3) & \multirow{2}{*}{21} & \multirow{2}{*}{0.28} & \multirow{2}{*}{51} & \multirow{2}{*}{0.68} & \multirow{2}{*}{0.38} & \multirow{2}{*}{0.50} & \multirow{2}{*}{43.75} & \multirow{2}{*}{$\begin{array}{l}{[57,60,70,} \\
86]\end{array}$} \\
\hline & & & & & powder & $\operatorname{Tr}$ & Wounds (NI 4) & & & & & & & & \\
\hline \multirow[b]{2}{*}{63} & \multirow{2}{*}{ Rosaceae } & \multirow{2}{*}{$\begin{array}{c}\text { Rosa indica } \mathrm{L} . / \mathrm{KD}- \\
102\end{array}$} & \multirow{2}{*}{ Gulab } & & \begin{tabular}{|c|}
$\begin{array}{c}\text { Soaked in water } \\
\text { overnight } \\
\text { (Infusion/Arq- } \\
\text { e-Guhlab) }\end{array}$ \\
\end{tabular} & $\mathrm{Tr} / \mathrm{Or}$ & $\begin{array}{c}\text { Eye cleaner (NI 12), heart tonic (NI } \\
7 \text { ) }\end{array}$ & \multirow{2}{*}{29} & \multirow{2}{*}{0.39} & \multirow{2}{*}{46} & \multirow{2}{*}{0.61} & \multirow{2}{*}{0.38} & \multirow{2}{*}{0.50} & \multirow{2}{*}{43.75} & \multirow[b]{2}{*}[61,72]{} \\
\hline & & & & & \begin{tabular}{|c|} 
Crushed in \\
sugar and kept \\
few days under \\
moonlight \\
\end{tabular} & Or & Constipation (NI 10) & & & & & & & & \\
\hline \multirow[b]{2}{*}{64} & \multirow[b]{2}{*}{ Rosaceae } & \multirow{2}{*}{$\begin{array}{l}\text { Rosa brunonii } \\
\text { Lindl./ KD- } 173\end{array}$} & \multirow[b]{2}{*}{ Tarnari } & & $\begin{array}{l}\text { Powder, } \\
\text { decoction }\end{array}$ & $\operatorname{Tr}$ & $\begin{array}{l}\text { Wounds (NI 7), infections(NI 11), } \\
\text { constipation (NI 9) }\end{array}$ & \multirow[b]{2}{*}{35} & \multirow[b]{2}{*}{0.47} & \multirow[b]{2}{*}{56} & \multirow[b]{2}{*}{0.75} & \multirow[b]{2}{*}{0.50} & \multirow[b]{2}{*}{0.33} & \multirow[b]{2}{*}{25.00} & \multirow[b]{2}{*}[60,67,87]{} \\
\hline & & & & & Powder & Or & *General weakness (NI 8) & & & & & & & & \\
\hline & & & & & Paste & $\operatorname{Tr}$ & Wounds (NI 8) & & & & & & & & \\
\hline 65 & Rosaceae & $\begin{array}{l}\text { Rubus ellipticus } \\
\text { Smith./ KD-124 }\end{array}$ & Aakhra & & $\begin{array}{l}\text { Crushed in } \\
\text { water (Juice), }\end{array}$ & Or & $\begin{array}{c}\text { *Cool demulcent (NI 9), } \\
\text { vaginal/seminal discharge (NI 6), } \\
\text { fever (NI 3), colic (NI 8), coughs (NI } \\
\text { 6), sore throat (NI 5). }\end{array}$ & 45 & 0.60 & 53 & 0.71 & 0.88 & 1.00 & 93.75 & $\begin{array}{c}{[57,61,70]} \\
{[72]}\end{array}$ \\
\hline 66 & Rubiaceae & $\begin{array}{c}\text { Rubia } \\
\text { codifoliaRoxb. ex } \\
\text { Desv./ KD-109 } \\
\end{array}$ & & hoce & $\begin{array}{l}\text { Crushed in } \\
\text { water (Juice) }\end{array}$ & Or & $\begin{array}{l}\text { *Amenorrhea (NI 12), dysentery (5) } \\
\text { intestinal worms in animals (2) }\end{array}$ & 19 & 0.25 & 32 & 0.43 & 0.38 & 0.50 & 43.75 & [53] \\
\hline
\end{tabular}




\begin{tabular}{|c|c|c|c|c|c|c|c|c|c|c|c|c|c|c|c|}
\hline Sr.\# & Family & \begin{tabular}{|c|} 
Plant species/ \\
Specimen number
\end{tabular} & Local name & ${ }^{A}$ Part used & ${ }^{B}$ Preparation & ${ }^{\mathrm{c}}$ Application & ${ }^{D}$ Properties/Aliment treated & ${ }^{\mathrm{E}} \mathbf{F C}$ & ${ }^{\mathrm{F}} \mathrm{RFC}$ & ${ }^{\mathrm{G}} \mathbf{U i}$ & ${ }^{\mathrm{H}} \mathbf{U V}$ & ${ }^{\text {IRel. PH }}$ & ${ }^{J}$ Rel. BS & ${ }^{\mathrm{K}} \mathbf{R I}$ & Literature \\
\hline 67 & Rutaceae & $\begin{array}{l}\begin{array}{c}\text { Zanthoxylum } \\
\text { alatum } \text { Roxb./ KD- } \\
356\end{array} \\
\quad\end{array}$ & Timmber & \% & $\begin{array}{c}\text { Kept on hand } \\
\text { (Asa), powder } \\
\text { mixed with } \\
\text { Mentha spp. } \\
\text { and table salt } \\
\text { eaten with } \\
\text { boiled egg, } \\
\text { decoction }\end{array}$ & $\operatorname{Tr}$ & $\begin{array}{c}\text { Piles (NI 9), chest pain ( NI 7), } \\
\text { *digestive problems (NI 9), fever (NI } \\
\text { 5), dyspepsia (NI 6), cholera (NI 8), } \\
\text { toothache (NI 6), rheumatism (NI 4) }\end{array}$ & 54 & 0.72 & 64 & 0.85 & 1.00 & 0.67 & 83.33 & $\begin{array}{c}{[10,61,68,} \\
72]\end{array}$ \\
\hline 68 & Rutaceae & \begin{tabular}{|} 
Citrus medica var. \\
acida Brandis/ KD- \\
246
\end{tabular} & Girgal & & $\begin{array}{c}\text { Juice mixed } \\
\text { with the } \\
\text { infusion of } \\
\text { Rosa indica and } \\
\text { glycerin }\end{array}$ & Or & $\begin{array}{l}\text { Softening of skin (NI 15), pain (NI } \\
\text { 7), heart tonic (NI 5), dysentery (NI } \\
\text { 2), antibiotic (NI 4) }\end{array}$ & 33 & 0.44 & 35 & 0.47 & 0.63 & 0.83 & 72.92 & {$[61,88]$} \\
\hline 69 & Sapindaceae & $\begin{array}{l}\text { Dodonaea } \\
\text { viscosa }(\text { L. }) \text { Jacq./ } \\
\text { KD-100 }\end{array}$ & Sanatha & & Exude, extracts & $\operatorname{Tr}$ & $\begin{array}{c}\text { Sore throat (NI 8), *diabetes (NI 11), } \\
\text { pain (NI 9), toothache (NI 7), wound } \\
\text { (NI 8) }\end{array}$ & 43 & 0.57 & 58 & 0.77 & 0.63 & 0.67 & 64.58 & $\begin{array}{c}{[10,48,60,} \\
61]\end{array}$ \\
\hline 70 & Sapindaceae & \begin{tabular}{|c|} 
Sapindus mukorossi \\
Gaertn. de Fruct./ \\
KD-132
\end{tabular} & Raintha & & Paste & $\operatorname{Tr}$ & $\begin{array}{c}\text { *Snake/ scorpion bites (NI15), } \\
\text { allergy (NI 7), skin freckles (NI 4), } \\
\text { inflammation (NI 6), anti-lice (NI 8), } \\
\text { dandruff (NI 9) }\end{array}$ & 49 & 0.65 & 55 & 0.73 & 0.75 & 0.33 & 54.17 & {$[10,70,79]$} \\
\hline 71 & Scrophulariaceae & \begin{tabular}{|} 
Verbascum thapsus \\
L./ KD-200
\end{tabular} & $\begin{array}{c}\text { Giddar } \\
\text { tammako }\end{array}$ & & Paste, smoked & $\operatorname{Tr}$ & $\begin{array}{l}\text { *Joint pain (NI 5), chest complaints } \\
\text { (NI 4), asthma (NI 2) }\end{array}$ & 11 & 0.15 & 22 & 0.29 & 0.38 & 0.33 & 35.42 & {$[46,72,89]$} \\
\hline 72 & Solanaceae & \begin{tabular}{|c|} 
Capsicum \\
frutescens var. \\
acuminate Fingerh/ \\
KD-301 \\
\end{tabular} & Mirch & & $\begin{array}{l}\text { Eaten with } \\
\text { bread }\end{array}$ & Or & $\begin{array}{l}\text { *Abdominal worms (NI 5), stomach } \\
\text { pain (NI } 9 \text { ), back pain (NI 7) }\end{array}$ & 21 & 0.28 & 43 & 0.57 & 0.38 & 0.33 & 35.42 & [89] \\
\hline \multirow[t]{2}{*}{73} & \multirow[t]{2}{*}{ Solanaceae } & \multirow[t]{2}{*}{$\begin{array}{l}\text { Solanum incanum } \\
\mathrm{L} . / \mathrm{KD}-26\end{array}$} & \multirow[t]{2}{*}{ Mohkari } & $\therefore$ & Paste/ Smoked & $\operatorname{Tr}$ & Toothache (NI 6), snake bite (NI 4) & \multirow[t]{2}{*}{17} & \multirow[t]{2}{*}{0.23} & \multirow[t]{2}{*}{38} & \multirow[t]{2}{*}{0.53} & \multirow[t]{2}{*}{0.50} & \multirow[t]{2}{*}{0.67} & \multirow[t]{2}{*}{58.33} & \multirow[t]{2}{*}{ [51] } \\
\hline & & & & hot & Juice & Or & Pneumonia (NI 4), sore throat (NI 3) & & & & & & & & \\
\hline 74 & Solanaceae & $\begin{array}{c}\text { Solanum nigrum } \mathrm{L} . \\
/ \mathrm{KD}-69\end{array}$ & Kachach & 0 & Potherb/ juice & Or & $\begin{array}{l}\text { Heart disease (NI 15), fever (NI 8), } \\
\text { pain (NI 3) }\end{array}$ & 26 & 0.35 & 35 & 0.47 & 0.38 & 0.50 & 43.75 & {$[47,50]$} \\
\hline
\end{tabular}




\begin{tabular}{|c|c|c|c|c|c|c|c|c|c|c|c|c|c|c|c|}
\hline Sr.\# & Family & $\begin{array}{c}\text { Plant species/ } \\
\text { Specimen number }\end{array}$ & Local name & ${ }^{A}$ Part used & ${ }^{\text {BPreparation }}$ & ${ }^{{ }^{c} \text { Application }}$ & ${ }^{D}$ Properties/Aliment treated & ${ }^{\mathrm{E}} \mathbf{F C}$ & ${ }^{\mathrm{F}} \mathrm{RFC}$ & ${ }^{{ }^{6}} \mathbf{U i}$ & ${ }^{\mathrm{H}} \mathbf{U V}$ & ${ }^{\mathrm{I}}$ Rel. PH & ${ }^{\mathrm{J}}$ Rel. BS & ${ }^{\mathrm{K}_{\mathbf{R I}}}$ & Literature \\
\hline \multirow[b]{2}{*}{75} & \multirow[b]{2}{*}{ Solanaceae } & \multirow{2}{*}{\begin{tabular}{|} 
Solanum surattense \\
Shord Wendl. / KD- \\
37
\end{tabular}} & \multirow[b]{2}{*}{ Mohkari } & & Paste, smoke & $\operatorname{Tr}$ & $\begin{array}{l}\text { Pain (NI 9), inflammation (NI 7), } \\
\text { piles (NI 8) }\end{array}$ & \multirow[b]{2}{*}{53} & \multirow[b]{2}{*}{0.71} & \multirow[b]{2}{*}{39} & \multirow[b]{2}{*}{0.52} & \multirow[b]{2}{*}{0.50} & \multirow[b]{2}{*}{0.50} & \multirow[b]{2}{*}{50.00} & \multirow[b]{2}{*}{ [47] } \\
\hline & & & & & \begin{tabular}{|c|} 
Cooked in milk \\
(Curry), \\
Decoction with \\
the stem of \\
Tinospora \\
cordifolia \\
\end{tabular} & Or & $\begin{array}{c}\text { Backache (NI 11), fever (NI 12), } \\
\text { phlegmatic Cough (NI 6) }\end{array}$ & & & & & & & & \\
\hline 76 & Ulmaceae & $\begin{array}{l}\text { Celtis australis } \\
\text { L./KD-67 }\end{array}$ & Khirk & & Paste, decoction & $\operatorname{Tr}$ & $\begin{array}{l}\text { *Smallpox (NI 15), anti-allergic (NI } \\
\text { 10), dysentery (NI 12), inter } \\
\text { menstrual bleeding (NI 7), bacterial } \\
\text { infection (NI5). }\end{array}$ & 49 & 0.65 & 61 & 0.81 & 0.75 & 1.00 & 87.50 & {$[60,69]$} \\
\hline 77 & Urticaceae & $\begin{array}{c}\text { Debregeasia } \\
\text { salicifolia } \text { (D. Don.) } \\
\text { Rendle./KD-96 }\end{array}$ & Sindari & & $\begin{array}{l}\text { Exude/paste } \\
\text { mixed with } \\
\text { mustard oil }\end{array}$ & $\operatorname{Tr}$ & $\begin{array}{l}\text { *Toothache (NI 7), skin rashes (NI } \\
\text { 4), infection (NI 10), eczema (NI 9) }\end{array}$ & 30 & 0.40 & 43 & 0.57 & 0.50 & 0.33 & 41.67 & {$[60,69,79]$} \\
\hline 78 & Verbenaceae & $\begin{array}{l}\text { Vitex negundo } \\
\text { Haussk./ KD-59 }\end{array}$ & Banah & & $\begin{array}{l}\text { Powder, } \\
\text { decoction }\end{array}$ & Or & $\begin{array}{l}\text { Abdominal pain (NI 8), dispersing } \\
\text { swellings of the joints from acute } \\
\text { rheumatism (NI 2), *toothache (NI } \\
\text { 12), ulcers (NI 4), flatulence,(NI 3) } \\
\text { fever (NI 3) }\end{array}$ & 32 & 0.43 & 53 & 0.71 & 0.75 & 0.50 & 62.50 & {$[90,91]$} \\
\hline 79 & Violaceae & $\begin{array}{c}\text { Viola } \\
\text { canescensWall. Ex } \\
\text { Roxb./ KD-43 }\end{array}$ & Banakhshah & & Decoction & Or & $\begin{array}{l}\text { Jaundice (NI 24), fever (NI 13), } \\
\text { gastrointestinal disorder (NI 8), } \\
\text { cough (NI 9) }\end{array}$ & 52 & 0.69 & 47 & 0.63 & 0.50 & 0.50 & 50.00 & {$[10,82]$} \\
\hline 80 & Xanthorrhoeaceae & $\begin{array}{l}\text { Aloe vera (L.) } \\
\text { Burm.f./ KD-77 }\end{array}$ & Kunwaargandal & & Potherb & Or & $\begin{array}{c}\text { *Pain (NI 5),* inflammation (NI 8), } \\
\text { wound healing (NI 3), }\end{array}$ & 16 & 0.21 & 33 & 0.44 & 0.38 & 0.17 & 27.08 & {$[60,79,92]$} \\
\hline
\end{tabular}

FC = frequency of citation, RFC = relative frequency of citation, Rel. PH = relative pharmacological properties, Rel. $\mathrm{BS}=$ relative body system treated, RI = relative importance, $\mathrm{UV}=$ use value index, Or = oral, Tr= tropical. *Novel use

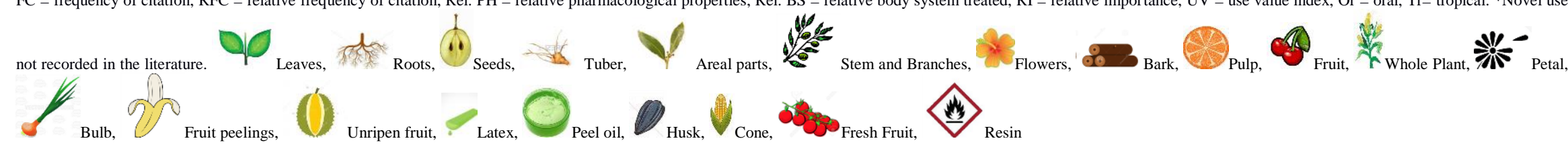

APPLIED ECOLOGY AND ENVIRONMENTAL RESEARCH 17(2):2799-2829.

http://www.aloki.hu • ISSN 15891623 (Print) • ISSN 17850037 (Online)

DOI: http://dx.doi.org/10.15666/aeer/1702_27992829

(c) 2019, ALÖKI Kft., Budapest, Hungary 


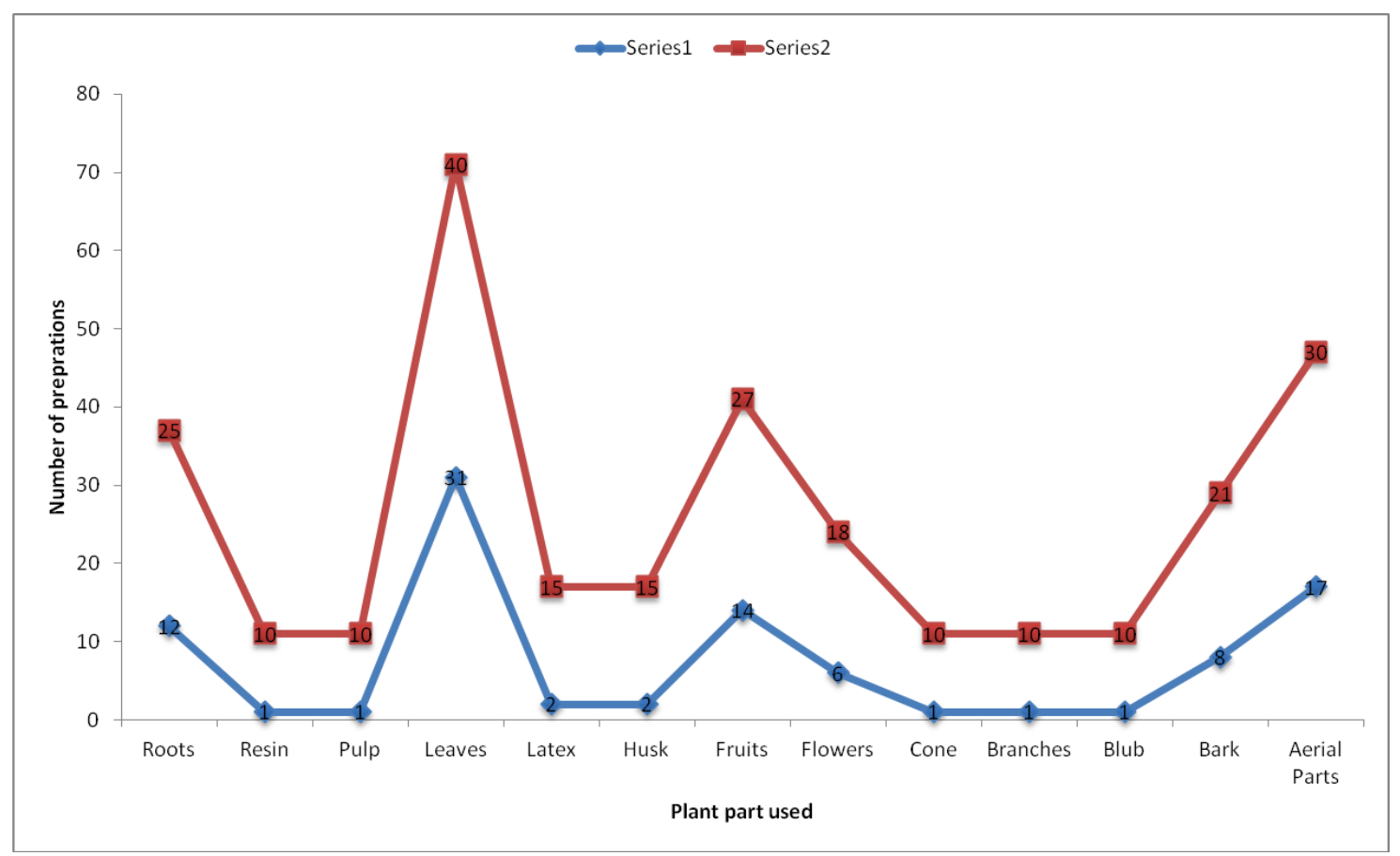

Figure 5. Different plant parts used in herbal preparations

\section{Methods of preparation and administration}

Different herbal recipes were prepared from the medicinal plants and method of preparation and application vary based on type of disease treated and actual site of application (Fig. 6). The most herbal recipes were prepared by grounding plant parts into powder about $16.4 \%$. Other methods of preparation and administration include: juice $(15 \%)$, paste $(14 \%)$, decoction $(11 \%)$, chewing $(6.3 \%)$, potherb $(5.7 \%)$, infusion $(3.8 \%)$, extract $(3.16 \%)$, boiling, smoking, poultice, latex and wrapping (2.53\% each), cooking and oil (1.9\% each), rubbing $(1.27 \%)$, herbal bath, smelling and tea $(0.63 \%$ each). Although most of the preparations were made from a single plant few were made from mixture of more than one plants, for example, seeds of Leucaena leucocephala were grounded and mixed with equal amount of powdered seeds of Albizia lebbeck to treat sexual debility. Similarly, roasted fruit of Papaver somniferum was ground with Terminalia chebula, Foeniculum vulgare and black salt for the treatment of abdominal pain and sleeplessness. With regard to the method of preparation, the undertaken study was in accordance with the work carried out by Mouterde (1983). Most of the preparations were administered orally $(57.58 \%)$, followed by tropical (39.62\%) and inhalation (2.8\%). These results were in accordance with Vijayakumar et al. (2015).

\section{Therapeutic indications}

\section{Informant's consensus factor $\left(F_{I C}\right)$}

In order to check the agreement of informants about the knowledge of plants uses, the recorded 90 diseases were grouped into 12 different disease categories according to IDC. The Informants Consensus Factor $\left(F_{I C}\right)$ values ranged from 0.80 to 0.91 , showed much homogeneity in nearly all the described disease categories (Table 3). Generally, FIC values range from 0 to 1 , in which the high values are good indicator of high 
informant consensus on the species used in the treatment of a particular category of illness (Heinrich et al., 1998; Trotter and Logan, 1986). The Informants Consensus Factor $\left(\mathrm{F}_{\mathrm{IC}}\right)$ analysis indicated that among the 12 disease categories skin and related

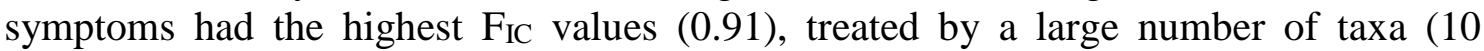
species) and 96 use reports. It was followed by circulatory problems (0.90), diabetes (0.90). The highest value of $F_{\text {IC }}$ suggests increased data about the usefulness of plants to cure a specific disease. The most commonly used plants to treat these diseases were Pinus roxburghii, Ranunculus arvensis Albizia lebbeck, Lepidium sativum, Chenopodium album, Otostegia limbata, Berberis lyceum, Medicago polymorpha and Adhatoda zeylanica. Although the FIC for gastrointestinal disorders (0.88) was less compared to some other ailment categories, however, a maximum number of plants (66 species) were recorded under this category. This category contains wide range of ailments such as abdominal pain, abdominal worms, piles, cholera, constipation, diarrhea, dysentery indigestion, flatulence, stomach disorder, intestinal problems, lack of appetite, nausea, vomiting and stomach pain (Afolayan et al., 2014; Abe and Ohtani, 2013). The plants with high FIC value might contain the high amount of bioactive compounds and can be subjected to further biochemical and pharmacological analysis.

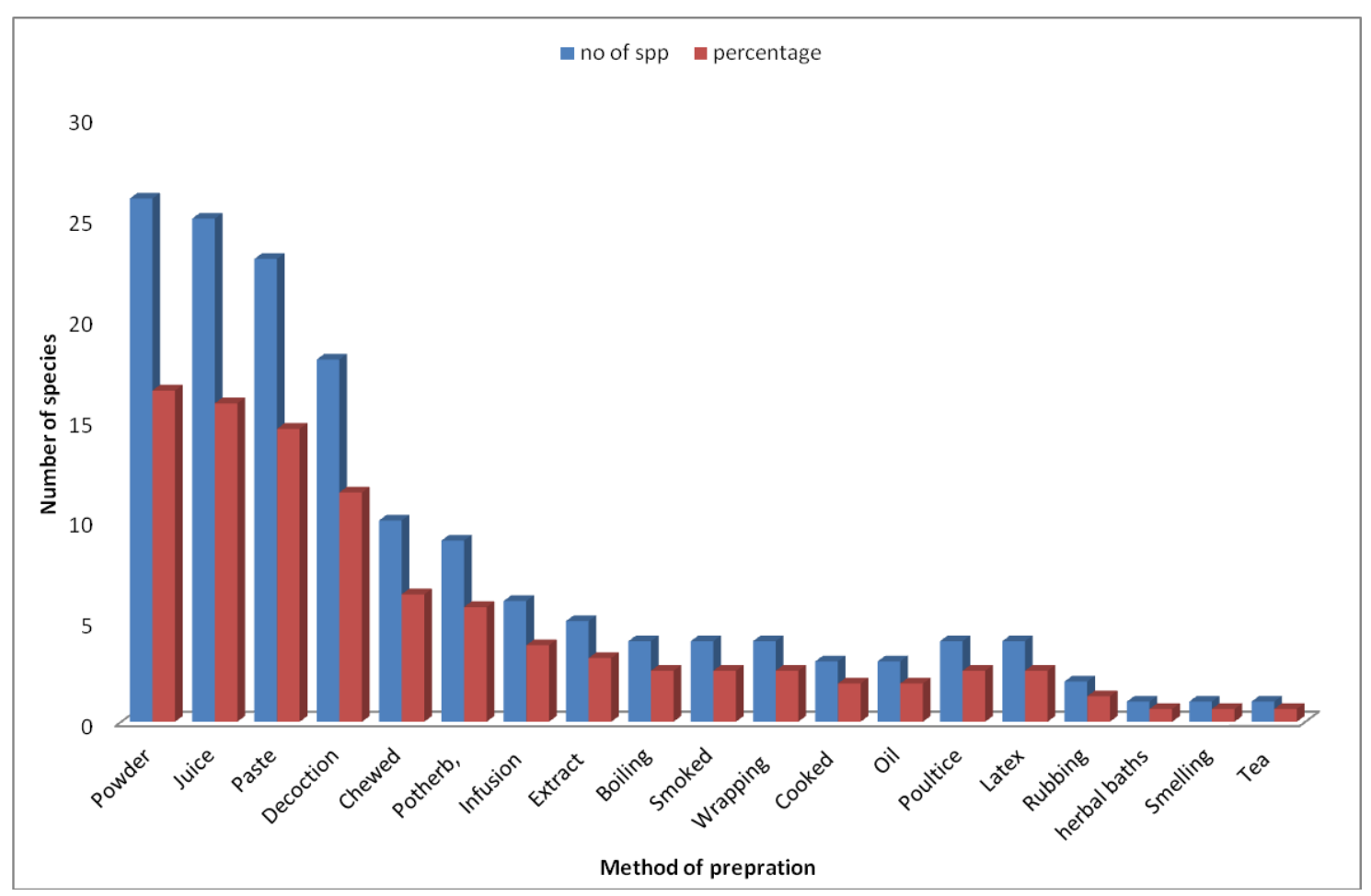

Figure 6. Various herbal preparations/formulations from the native flora of Rajh Mehal

\section{Medicinal importance (MI)}

The medicinal importance (MI) values can be used as a sign of the relative medicinal relevance of mentioned plant species (Table 3). These values can be used in finding variability among the pharmacological, environmental and cultural determinants between the investigated species and areas (Baydoun et al., 2015). The MI and FIC values were higher for skin and related symptoms (9.6) because weather conditions in study area quit severe dry and prolong winter; that is why people in the study area suffer 
skin problems very commonly. After skin diseases circulatory problems (8.93) were common, then diabetes (8.8) and digestive system problems, mouth, ear and oral disease (8.45). These values were closer to the findings of Baydoun et al. (2015) and higher than the MI values reported by Carrió and Vallès (2012).

Table 3. International classification of diseases (ICD) classification and disease categories along with various diseases versus reports, number of taxa, total reports and FIC

\begin{tabular}{|c|c|c|c|c|c|c|}
\hline Sr. no & Disease category & Symptoms & $\begin{array}{l}\text { No. of } \\
\text { taxa }\end{array}$ & $\begin{array}{c}\text { Use } \\
\text { reports }\end{array}$ & FIC & MI \\
\hline 1 & $\begin{array}{l}\text { Circulatory problems } \\
\text { (I00-I99) }\end{array}$ & $\begin{array}{c}\text { Anemia (10), blood pressure (20), blood } \\
\text { purifier (32), heart disease (15), heart disease } \\
\text { (29), edema (3), heart tonic (16) }\end{array}$ & 14 & 125 & 0.90 & 8.93 \\
\hline 2 & $\begin{array}{l}\text { Digestive system } \\
\text { problems (K00-K93), } \\
\quad(\text { R00-R99) }\end{array}$ & $\begin{array}{l}\text { Abdominal Pain (92), abdominal worms (5), } \\
\text { piles (45), cholera (8), constipation (87) } \\
\text { diarrhea 61 digestive problem (9) dysentery } \\
\text { (110) indigestion (37) flatulence } 18 \text { stomach } \\
\text { disorder (8) intestinal problems (12) lack of } \\
\text { appetite (15) nausea and vomiting (26) } \\
\text { stomach pain (24) scurvy (1) jaundice (94) }\end{array}$ & 66 & 558 & 0.88 & 8.45 \\
\hline 3 & $\begin{array}{l}\text { Respiratory System (J00- } \\
\text { J99) }\end{array}$ & $\begin{array}{l}\text { Asthma (45), bad breath (4), chest } \\
\text { complaints (4), chest pain (7), flue and cold } \\
\text { (26), cough (94) haemopty (7) pneumonia }\end{array}$ & 25 & 191 & 0.87 & 7.64 \\
\hline 4 & Diabetes (E10-E14) & Diabetes (88) & 10 & 88 & 0.90 & 8.8 \\
\hline 5 & $\begin{array}{l}\text { Musculoskeletal (M00- } \\
\text { M99) }\end{array}$ & $\begin{array}{l}\text { Back pain (43) body pain (7) joints pain (2) } \\
\text { inflammation (69) joint pain (43) pain (76) } \\
\text { pain and inflammation (22) swelling (33) } \\
\text { fractured bones (8), rheumatic pain (23) }\end{array}$ & 48 & 326 & 0.86 & 6.79 \\
\hline 6 & Infections (A00-A09) & $\begin{array}{l}\text { Bacterial infection (12), ear infection (13), } \\
\text { infection (40), skin infection (2), pus } \\
\text { formation (6), Fever (73) }\end{array}$ & 10 & 73 & 0.88 & 7.3 \\
\hline 7 & $\begin{array}{c}\text { Skin and related } \\
\text { symptoms (S00-T98) }\end{array}$ & $\begin{array}{l}\text { Boils (65), burns (9), measles/ mumps (2), } \\
\text { pimples (3), Small pox (7) }\end{array}$ & 10 & 96 & 0.91 & 9.6 \\
\hline 8 & $\begin{array}{l}\text { Dermatological problems } \\
\text { (L00-L99) }\end{array}$ & $\begin{array}{c}\text { Dermatitis (7), heel cracks (5), eczema (9), } \\
\text { ringworm (7), scabies (3), skin disease (4), } \\
\text { skin freckles (4), skin rashes (19), allergy } \\
\text { (17) athlete foot (5) }\end{array}$ & 13 & 60 & 0.80 & 5.8 \\
\hline 9 & $\begin{array}{l}\text { Others (Tonic, cool } \\
\text { demulcent drinks, } \\
\text { sedative }\end{array}$ & $\begin{array}{l}\text { Cool demulcent (25), hair tonic (4), hot tonic } \\
\text { (14), nerve tonic (11), refrigerant (6), tonic } \\
\text { (5), uterus tonic (5), tooth and gum tonic (4), } \\
\text { sedative (7), ulcers (4), heat stock (9) }\end{array}$ & 13 & 94 & 0.87 & 7.4 \\
\hline 10 & Antidotes T36-T50 & Dog-bite (9) Insect bite (15) snake bite (27) & 8 & 51 & 0.86 & 6.38 \\
\hline 11 & $\begin{array}{l}\text { Worm insect related } \\
\text { diseases }\end{array}$ & $\begin{array}{c}\text { Anthelmintic (14) antibiotic (4) anti-lice (12) } \\
\text { expel worms (5) intestinal worms (10) kill } \\
\text { worms (2) }\end{array}$ & 8 & 47 & 0.85 & 5.88 \\
\hline 12 & $\begin{array}{l}\text { Oral, dental, hair and } \\
\text { ENT }\end{array}$ & $\begin{array}{l}\text { Bleeding from nose (9) bleeding gums (5) } \\
\text { earache (41) tooth discoloration (16) eye } \\
\text { cleaner (16) weak eye sight (22) mouth } \\
\text { infection (21) mouth inflammation (11) } \\
\text { mouth ulcer (32), ophthalmia (8), toothache } \\
\text { (63), weakness (31), throat pain (38), } \\
\text { swollen gums (2), dandruff (14) hair fall } \\
\text { (17) headache (9), sore throat (16) }\end{array}$ & 66 & 558 & 0.88 & 8.45 \\
\hline
\end{tabular}

FIC $=$ informants' consensus factor, $\mathrm{MI}=$ medicinal importance 


\section{Relative importance (RI)}

The highest RI value was obtained for Adhatoda zeylanica (93.75) Zanthoxylum alatum (91.67), Berberis lyceum, Juglans regia (87.50 each) Punica granatum, Olea ferruginea (83.33 each), and Solanum surattense (79.17) which indicated that these plants possess strong pharmacological properties and are used more frequently in the study area. The important and most commonly cited plants belonged to Asteraceae, Lamiaceae, Solanacea and, Rosaceae. Theses finding agrees with the early reports by Carmen Juárez-Vázquez et al. (2013), Yaseen et al. (2015) and Alonso-Castro et al. (2012).

\section{Smith's salience analysis (SS)}

Salience analysis (Smith, 1993) accounted for frequency of mention; on an average 60 illnesses were listed. In free-listing we interview 112 informants but for Salience analysis and ranking of illness 10 most informative informants were chosen. The Table 4 shows salience estimates for each illness, indicating both the number of people who mentioned the illness and the order of their responses. In Smith's Salience analysis abdominal pain and worms had the highest overall salience index, mentioned by $73.21 \%$ of informants. Constipation was the second most salient illness had $70.54 \%$ of informants and Pain was the third most cited illness treated by the plants in the study had $67.8 \%$ frequency. Sleeplessness, uterus disease, weak eyesight and bleeding gonorrhea were the less salient diseases. Composite Salience was another step to reinforcement the results of Smith's Salience by narrow down the informant numbers, result was draw the on the base of ten key informants.

Table 4. Results of ANTHROPAC analysis of Illness free-lists and Smith's salience index along with composite salience

\begin{tabular}{|c|c|c|c|c|c|c|c|c|c|c|c|c|c|c|}
\hline \multirow{2}{*}{$\begin{array}{l}\text { S. } \\
\text { No }\end{array}$} & \multirow{2}{*}{ Illness } & \multirow{2}{*}{$\begin{array}{l}\text { Frequency } \\
(\%) \mathrm{N}=112\end{array}$} & \multicolumn{10}{|c|}{ Inverted rank/total listed = Smith's salience index } & \multirow{2}{*}{$\underset{\Sigma}{\text { Illness }}$} & \multirow{2}{*}{ 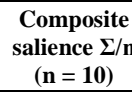 } \\
\hline & & & SS1 & SS2 & SS3 & SS4 & SS5 & SS6 & SS7 & SS8 & SS9 & SS10 & & \\
\hline 1 & $\begin{array}{c}\text { Abdominal } \\
\text { pain and } \\
\text { worms }\end{array}$ & $82(73.21)$ & $\begin{array}{c}60 / 60= \\
1\end{array}$ & & $\begin{array}{c}58 / 60= \\
0.96\end{array}$ & & & $\begin{array}{c}53 / 60= \\
0.883\end{array}$ & & & $\begin{array}{c}52 / 60= \\
0.867\end{array}$ & $\begin{array}{c}51 / 60= \\
0.850\end{array}$ & 4.56 & 0.4560 \\
\hline 2 & Constipation & $79(70.54)$ & & $\begin{array}{c}60 / 60= \\
1\end{array}$ & & & & & & \begin{tabular}{|c|}
$53 / 60=$ \\
0.883 \\
\end{tabular} & & & 1.883 & 0.1883 \\
\hline 3 & Pain & $76(67.86)$ & $\begin{array}{c}58 / 60= \\
0.96\end{array}$ & & & & & & & & & & 1.827 & 0.1827 \\
\hline 4 & $\begin{array}{c}\text { Sexual } \\
\text { dysfunction }\end{array}$ & $72(64.29)$ & & & & $\begin{array}{c}56 / 60= \\
0.933\end{array}$ & & & & & & & 0.933 & 0.0933 \\
\hline 5 & $\begin{array}{c}\text { Teeth } \\
\text { problems }\end{array}$ & $71(63.39)$ & $\begin{array}{c}56 / 60= \\
0.933\end{array}$ & $\begin{array}{c}58 / 60= \\
0.96\end{array}$ & & & & & & & & & 1.893 & 0.1893 \\
\hline 6 & Wounds & $70(62.50)$ & & & & & $\begin{array}{c}52 / 60= \\
0.867 \\
\end{array}$ & & & $\begin{array}{l}1 / 60= \\
0.017\end{array}$ & & & 0.867 & 0.0867 \\
\hline 7 & Jaundice & $69(61.61)$ & & & & & & & & & & & 0.883 & 0.0883 \\
\hline 8 & Skin problems & $69(61.61)$ & $\begin{array}{c}51 / 60= \\
0.850 \\
\end{array}$ & & & & & $\begin{array}{c}53 / 60= \\
0.883 \\
\end{array}$ & & & & & 0.85 & 0.0850 \\
\hline 9 & Eczema & $68(60.71)$ & & & & & & & & & & $\begin{array}{c}50 / 60= \\
0.833\end{array}$ & 0.833 & 0.0833 \\
\hline 10 & Cough & $67(59.82)$ & $\begin{array}{c}50 / 60= \\
0.833 \\
\end{array}$ & & & & & & $\begin{array}{l}1 / 60= \\
0.017\end{array}$ & & & & 0.833 & 0.0833 \\
\hline 11 & Diabetes & $67(59.82)$ & & $\begin{array}{l}1 / 60= \\
0.017\end{array}$ & $\begin{array}{c}48 / 60= \\
0.800\end{array}$ & & & & & & & & 0.8 & 0.0800 \\
\hline 12 & Diarrhea & $67(59.82)$ & \begin{tabular}{|c|}
$46 / 60=$ \\
0.767 \\
\end{tabular} & & & & $\begin{array}{l}1 / 60= \\
0.017\end{array}$ & & & & & & 0.767 & 0.0767 \\
\hline 13 & Blood purifier & $66(58.93)$ & & & & $\begin{array}{c}46 / 60= \\
0.768\end{array}$ & & & $\begin{array}{l}1 / 60= \\
0.017\end{array}$ & & & $\begin{array}{l}1 / 60= \\
0.017\end{array}$ & 0.768 & 0.0768 \\
\hline 14 & Cooling agent & $66(58.93)$ & $\begin{array}{c}46 / 60= \\
0.769\end{array}$ & & & & $\begin{array}{l}5 / 60= \\
0.083\end{array}$ & & & $\begin{array}{c}46 / 60= \\
0.770\end{array}$ & & & 1.539 & 0.1539 \\
\hline 15 & Dysentery & $65(58.04)$ & & $\begin{array}{l}1 / 60= \\
0.017\end{array}$ & & & & $\begin{array}{c}46 / 60= \\
0.770\end{array}$ & & & $\begin{array}{l}1 / 60= \\
0.017\end{array}$ & & 0.77 & 0.0770 \\
\hline
\end{tabular}




\begin{tabular}{|c|c|c|c|c|c|c|c|c|c|c|c|c|c|c|}
\hline \multirow{2}{*}{$\begin{array}{l}\text { S. } \\
\text { No }\end{array}$} & \multirow{2}{*}{ Illness } & \multirow{2}{*}{$\begin{array}{l}\text { Frequency } \\
(\%) \mathbf{N}=112\end{array}$} & \multicolumn{10}{|c|}{ Inverted rank/total listed = Smith's salience index } & \multirow{2}{*}{$\underset{\Sigma}{\text { Illness }}$} & \multirow{2}{*}{$\begin{array}{c}\text { Composite } \\
\text { salience } \Sigma / n \\
(n=10)\end{array}$} \\
\hline & & & SS1 & SS2 & SS3 & SS4 & SS5 & SS6 & SS7 & SS8 & SS9 & SS10 & & \\
\hline 16 & Joints pain & $65(58.04)$ & \begin{tabular}{|c|}
$44 / 60=$ \\
0.733
\end{tabular} & & & & & & & & & & 0.733 & 0.0733 \\
\hline 17 & Backpain & 65 (58.04) & & & \begin{tabular}{|c|}
$44 / 60=$ \\
0.734
\end{tabular} & & $\begin{array}{l}9 / 60= \\
0.150\end{array}$ & & & & & & 0.734 & 0.0734 \\
\hline 18 & Boils & $64(57.14)$ & \begin{tabular}{|c|}
$44 / 60=$ \\
0.735 \\
\end{tabular} & & & $\begin{array}{l}9 / 60= \\
0.150\end{array}$ & & & & $\begin{array}{l}1 / 60= \\
0.017\end{array}$ & & & 0.735 & 0.0735 \\
\hline 19 & $\begin{array}{c}\text { Gastrointestina } \\
\text { 1 disorder }\end{array}$ & 64 (57.14) & & & $\begin{array}{l}9 / 60= \\
0.150\end{array}$ & & & $\begin{array}{c}43 / 60= \\
0.717\end{array}$ & & & & & 0.717 & 0.0717 \\
\hline 20 & \begin{tabular}{c|c|} 
Mouth \\
infection
\end{tabular} & $63(56.25)$ & & & & & & & $\begin{array}{l}3 / 60= \\
0.050\end{array}$ & & & $\begin{array}{c}42 / 60= \\
0.700 \\
\end{array}$ & 0.7 & 0.0700 \\
\hline 21 & $\begin{array}{c}\text { Nausea and } \\
\text { vomiting }\end{array}$ & $63(56.25)$ & \begin{tabular}{|c|}
$42 / 60=$ \\
0.701 \\
\end{tabular} & & & $\begin{array}{c}42 / 60= \\
0.700\end{array}$ & & & & & & & 1.401 & 0.1401 \\
\hline 22 & $\begin{array}{c}\text { Throat } \\
\text { problems }\end{array}$ & $62(55.36)$ & & & \begin{tabular}{|c|}
$41 / 60=$ \\
0.683
\end{tabular} & & & & & $\begin{array}{l}42 / 60= \\
0.700\end{array}$ & & & 1.383 & 0.1383 \\
\hline 23 & Weakness & $61(54.46)$ & \begin{tabular}{|c|}
$39 / 60=$ \\
0.650
\end{tabular} & & & & & & \begin{tabular}{|c|}
$42 / 60=$ \\
0.700
\end{tabular} & & & & 1.35 & 0.1350 \\
\hline 24 & $\begin{array}{c}\text { Animal or } \\
\text { insect bites }\end{array}$ & $60(53.57)$ & & & & $\begin{array}{c}39 / 60= \\
0.651\end{array}$ & & & & & $\begin{array}{c}1 / 60=0 . \\
017\end{array}$ & & 0.651 & 0.0651 \\
\hline 25 & Ear pain & $60(53.57)$ & & & & & $\begin{array}{c}42 / 60= \\
0.700\end{array}$ & & & & & $\begin{array}{c}39 / 60= \\
0.652\end{array}$ & 1.352 & 0.1352 \\
\hline 26 & Flatulence & $59(52.68)$ & & & \begin{tabular}{|c|}
$37 / 60=$ \\
0.617
\end{tabular} & & & & & $\begin{array}{c}42 / 60= \\
0.700\end{array}$ & & & 1.317 & 0.1317 \\
\hline 27 & Hair fall & $58(51.79)$ & \begin{tabular}{|c|}
$36 / 60=$ \\
0.600
\end{tabular} & & & & & & & & & & 0.6 & 0.0600 \\
\hline 28 & Indigestion & $57(50.89)$ & & & & $\begin{array}{c}34 / 60= \\
0.567\end{array}$ & & & $\begin{array}{l}1 / 60= \\
0.017\end{array}$ & & & & 0.567 & 0.0567 \\
\hline 29 & Inflammation & $56(50.0)$ & & $\begin{array}{c}33 / 60= \\
0.550\end{array}$ & & & & & & & $\begin{array}{l}1 / 60= \\
0.017\end{array}$ & & 0.55 & 0.0550 \\
\hline 30 & Piles & $55(49.11)$ & & & $\begin{array}{c}31 / 60= \\
0.517\end{array}$ & & & $\begin{array}{l}1 / 60= \\
0.017\end{array}$ & & & & & 0.517 & 0.0517 \\
\hline 31 & Swellings & $54(48.21)$ & $\begin{array}{c}30 / 60= \\
0.500\end{array}$ & & & & & & & & & $\begin{array}{l}1 / 60= \\
0.017\end{array}$ & 0.5 & 0.0500 \\
\hline 32 & $\begin{array}{l}\text { Urinary } \\
\text { diseases }\end{array}$ & $53(47.32)$ & & & & $\begin{array}{c}27 / 60= \\
0.450\end{array}$ & & & $\begin{array}{l}5 / 60= \\
0.083\end{array}$ & & & & 0.45 & 0.0450 \\
\hline 33 & Cold and flu & $52(46.43)$ & \begin{tabular}{|c|}
$26 / 60=$ \\
0.433 \\
\end{tabular} & & & & & & & & & & 0.433 & 0.0433 \\
\hline 34 & Eye cleaner & $51(45.54)$ & & & \begin{tabular}{|c|}
$24 / 60=$ \\
0.400
\end{tabular} & & & $\begin{array}{l}5 / 60= \\
0.083\end{array}$ & & & & & 0.4 & 0.0400 \\
\hline 35 & Heart tonic & $50(44.64)$ & $\begin{array}{c}22 / 60= \\
0.367\end{array}$ & & & & & & & & $\begin{array}{l}5 / 60= \\
0.083\end{array}$ & & 0.367 & 0.0367 \\
\hline 36 & Pimples & $49(43.75)$ & & & & $\begin{array}{c}20 / 60= \\
0.333\end{array}$ & & & & $\begin{array}{l}3 / 60= \\
0.050\end{array}$ & & & 0.333 & 0.0333 \\
\hline 37 & Abortion & $48(42.86)$ & $\begin{array}{c}18 / 60= \\
0.300\end{array}$ & & & & & & & & & & 0.3 & 0.0300 \\
\hline 38 & Amenorrhea & $47(41.96)$ & & & $\begin{array}{c}17 / 60= \\
0.283 \\
\end{array}$ & & & & & & $\begin{array}{l}3 / 60= \\
0.050\end{array}$ & & 0.283 & 0.0283 \\
\hline 39 & Anorexia & $46(41.07)$ & \begin{tabular}{|c|}
$17 / 60=$ \\
0.284
\end{tabular} & & & & & & & & & $\begin{array}{l}2 / 60= \\
0.033\end{array}$ & 0.284 & 0.0284 \\
\hline 40 & Antilice & $46(40.18)$ & & & $\begin{array}{l}3 / 60= \\
0.050\end{array}$ & & & & $\begin{array}{c}15 / 60= \\
0.250\end{array}$ & & & & 0.25 & 0.0250 \\
\hline 41 & Asthma & $45(40.18)$ & \begin{tabular}{|c|}
$12 / 60=$ \\
0.200
\end{tabular} & & & & & $\begin{array}{l}3 / 60= \\
0.050\end{array}$ & & & & & 0.293 & 0.0293 \\
\hline 42 & Athlete foot & $41(36.61)$ & & $\begin{array}{l}4 / 60= \\
0.067\end{array}$ & & $\begin{array}{c}11 / 60= \\
0.183\end{array}$ & & & & & & & 0.25 & 0.0250 \\
\hline 43 & Blood pressure & $39(34.82)$ & $\begin{array}{c}10 / 60= \\
0.167\end{array}$ & & & & & & & & & & 0.567 & 0.0567 \\
\hline 44 & Dandruff & $36(42.14)$ & & & $\begin{array}{l}9 / 60= \\
0.150\end{array}$ & & & $\begin{array}{l}1 / 60= \\
0.017\end{array}$ & & & & & 0.167 & 0.0167 \\
\hline 45 & Edema & $32(28.57)$ & $\begin{array}{l}9 / 60= \\
0.151\end{array}$ & & & & & & & & $\begin{array}{l}1 / 60= \\
0.017\end{array}$ & & 0.168 & 0.0168 \\
\hline 46 & Hot tonic & $30(26.79)$ & & & & & $\begin{array}{l}1 / 60= \\
0.017\end{array}$ & & & $\begin{array}{l}9 / 60= \\
0.152\end{array}$ & & & 0.169 & 0.0169 \\
\hline 47 & Infection & $30(26.79)$ & $\begin{array}{l}7 / 60= \\
0.117\end{array}$ & & $\begin{array}{l}1 / 60= \\
0.017\end{array}$ & & & & $\begin{array}{l}1 / 60= \\
0.017\end{array}$ & & & & 0.151 & 0.0151 \\
\hline 48 & Injury & $25(22.32)$ & & $\begin{array}{l}6 / 60= \\
0.100\end{array}$ & & & & $\begin{array}{l}2 / 60= \\
0.033\end{array}$ & & & & & 0.133 & 0.0133 \\
\hline 49 & Leucorrhoea & $23(19.64)$ & $\begin{array}{l}6 / 60= \\
0.101\end{array}$ & & & & & & $\begin{array}{c}3 / 60=0 . \\
050\end{array}$ & & & & 0.151 & 0.0151 \\
\hline 50 & Nerve tonic & $22(19.64)$ & & & & $\begin{array}{l}6 / 60= \\
0.102\end{array}$ & & & & & $\begin{array}{l}1 / 60= \\
0.017\end{array}$ & & 0.119 & 0.0119 \\
\hline 51 & Ophthalmia & $20(17.86)$ & & & & & $\begin{array}{c}27 / 60= \\
0.450\end{array}$ & & & $\begin{array}{l}5 / 60= \\
0.083\end{array}$ & & & 0.45 & 0.0450 \\
\hline
\end{tabular}




\begin{tabular}{|c|c|c|c|c|c|c|c|c|c|c|c|c|c|c|}
\hline \multirow{2}{*}{$\begin{array}{l}\text { S. } \\
\text { No }\end{array}$} & \multirow{2}{*}{ Illness } & \multirow{2}{*}{$\begin{array}{l}\text { Frequency } \\
(\%) \mathrm{N}=112\end{array}$} & \multicolumn{10}{|c|}{ Inverted rank/total listed = Smith's salience index } & \multirow{2}{*}{$\begin{array}{c}\text { Illness } \\
\Sigma\end{array}$} & \multirow{2}{*}{$\begin{array}{c}\text { Composite } \\
\text { salience } \Sigma / \mathbf{n} \\
(\mathbf{n}=10)\end{array}$} \\
\hline & & & SS1 & SS2 & SS3 & SS4 & SS5 & SS6 & SS7 & SS8 & SS9 & SS10 & & \\
\hline 52 & Pneumonia & $15(13.39)$ & & & $\begin{array}{l}1 / 60= \\
0.017\end{array}$ & & & & & & & $\begin{array}{l}5 / 60= \\
0.084\end{array}$ & 0.101 & 0.0101 \\
\hline 53 & Pus formation & $14(12.50)$ & & & & $\begin{array}{l}4 / 60= \\
0.067\end{array}$ & & & & & & & 0.067 & 0.0067 \\
\hline 54 & Ringworm & $13(11.61)$ & & & & & & $\begin{array}{l}4 / 60= \\
0.067\end{array}$ & & & & & 0.067 & 0.0067 \\
\hline 55 & Sedative & $12(10.71)$ & $\begin{array}{l}3 / 60= \\
0.050\end{array}$ & & & & & & & & & & 0.05 & 0.0050 \\
\hline 56 & Sleeplessness & $10(8.93)$ & & & $\begin{array}{l}3 / 60= \\
0.050\end{array}$ & & & & & & & & 0.05 & 0.0050 \\
\hline 57 & Uterus disease & $10(8.93)$ & $\begin{array}{l}2 / 60= \\
0.033\end{array}$ & & & & & & & & & & 0.033 & 0.0033 \\
\hline 58 & $\begin{array}{c}\text { Weak eye } \\
\text { sight }\end{array}$ & $9(8.04)$ & & & & & & & $\begin{array}{l}2 / 60= \\
0.033\end{array}$ & & & & 0.033 & 0.0033 \\
\hline 59 & Bleeding & $5(4.46)$ & & & & & & & & & & $\begin{array}{l}1 / 60= \\
0.017\end{array}$ & 0.017 & 0.0017 \\
\hline 60 & Gonorrhea & $3(2.68)$ & $\begin{array}{c}1 / 60= \\
0.017\end{array}$ & & & & & & & & & & 0.017 & 0.0017 \\
\hline
\end{tabular}

\section{Use value index $(U V)$ and relative frequency of citation (RFC)}

The use value (UV) shows the range to which a species can be used and the species with high UV values were mostly exploited in the area to cure a specific aliment. Zanthoxylum alatum (1.19), Olea ferruginea (1.11), Solanum surattense (1.05) and Juglans regia (1.04) possessed the higher use values. Table 2 showed high values of $\mathrm{UV}$ and RFC of different plant species. It is evident from the results that Adhatoda zeylanica, (0.79) Berberis lyceum (0.77), Juglans regia (0.75) and, Punica granatum var. nana (0.73) and Zanthoxylum alatum (0.72) were the most cited species in the research area. On the bases of RFC and UV we concluded, since these species are very commonly available in the area, therefore the inhabitants mostly cited these. Moreover, these species were native to that area and were present for decades, so their properties to cure different disease got popularized among the indigenous peoples. A strong significant and positive correlation $\left(\mathrm{r}^{2}=0.60\right)$ was recorded between UV and RFC showing that by increase in number of informants the knowledge about plant use in herbal medicine will also increase. These results indicate that the study can make a significant contribution to folk knowledge on the use of medicinal plants and further laboratory-based investigations could help in identifying the active ingredients of the most commonly exploited plants. The variation in data can be explained by coefficient of determination and in present study the value of $\mathrm{R}^{2}$ was 0.36 reflecting that $33 \%$ variation in use value can be explained in terms of relative frequency of citation (Table 5).

Table 5. Pearson correlation between $U V$ and RFC

\begin{tabular}{c|c|c|c|c}
\hline \multicolumn{2}{c|}{} & UV & RFC & Mean \pm std. deviation \\
\hline \multirow{2}{*}{ UV } & Pearson correlation & 1 & $0.606^{* *}$ & \\
& Sig. (2-tailed) & & 0.000 & $0.6034 \pm 0.23249$ \\
& $\mathrm{~N}$ & 80 & 80 & \\
\hline \multirow{2}{*}{ RFC } & Pearson correlation & $0.606^{* *}$ & 1 & \\
& Sig. (2-tailed) & 0.000 & & $0.4250 \pm 0.19266$ \\
& $\mathrm{~N}$ & 80 & 80 & \\
\hline
\end{tabular}

$r^{2}=0.36$ 


\section{Discussion}

This study documented the good diversity of medicinal plants coupled with their therapeutic use and medicinal importance in the indigenous communities of Rajh Mehal, AJK. The ethnobotanical inventory consists of an ample therapeutic indication of 80 medicinal plants distributed in 12 disease categories that revealed a good picture of the health disorders of the native population. The natives mostly prefer single species based preparations instead of mixtures. The high Informant Consensus (FIC) suggests that current use and knowledge of medicinal plants are still strong and local inhabitants have a high dependency on medicinal plants in meeting their primary health care. Many novel uses of plants were documented after comparing with the previously published literature. Most of the documented species possessed similar uses; however, 16 species possessed various novel uses which were not previously recorded. These include: Conyza bonariensis (Wound), Carthamus tinctorius (Pneumonia), Celtis australis (Smallpox, anti-allergic), Dodonaea viscosa (Toothache, diabetes), Ipomoea carnea (Athlete foot), Launaea taraxacifolia (Diabetes, pain), Leucaena leucocephala (Sexual debility), Olea ferruginea (Mouth ulcer, throat pain), Pinus roxburghii (Diabetes), Plectranthus rugosus (Mouth infection), Rosa brunonii (General weakness), Rubia cordifolia (Amenorrhea), Rubus ellipticus (Cool demulcent), Rumex nepalensis (Edema, weakness), Thalictrum aquilegifolium (Sunstroke) and Zizyphus oxyphylla (Fever, abdominal pain). In Smith's Salience analysis indicated that abdominal pain and worms had the highest overall salience index, mentioned by $73.21 \%$ informants. Constipation was the second most salient illness had $70.54 \%$ of informants and Pain was the third most cited illness treated by the plants in the study had $67.8 \%$ frequency but Sleeplessness, uterus disease, weak eyesight and bleeding gonorrhea were the less salient diseases. Composite Salience was another step to reinforcement the results of Smith's Salience by narrow down the informant numbers, result was draw the on the base of ten key informants. On the bases of RFC and UV we can say that, these species are very commonly available in the area, therefore the inhabitants mostly cited these. Moreover, these species were native to that area and were present for decades, so their properties to cure different disease got popularized among the indigenous peoples.

\section{Conclusion}

The present study can serve as best baseline study to search active ingredients of plants which are being utilized as herbal medicine in the study area, particularly plants with novel uses. These plants might be subjected to further lab and clinical analysis for validation of results from present study. This traditional knowledge together with scientific studies can lead to powerful discovery for newer, safer and affordable medicines. Furthermore, this study could be helpful in the conservation of the traditional knowledge of medicinal plants of the remote areas like Rajh Mehal for the uplift of a good life standard and livelihood of the local communities is indispensable. High dependency of local communities on local flora is resulting in extinction of medicinal plants, so step should be taken for conservation and management of these valuable plant resources of this territory.

Acknowledgements. We are enormously thankful to the local inhabitants/informants of the study area for their assistance and knowledge sharing, without which such study is unfeasible. I want to acknowledge 
COMSATS Institute of Information Technology, Islamabad, English language department for language editing.

Author's contributions. MFQ and RQ designed experiments, field data were collected by QFM, MSA and WA, analytic analysis was also done by QFM and AS, HS, MFQ wrote first draft of manuscript, RQ reviewed and made amendments in original draft of manuscript.

Conflict of interests. We declare that we have no conflict of interests.

Ethics approval. Before initiating of our survey ethical approval for the study was obtained from the COMSATS Institute of Information Technology Ethics Committee. Legal permission was taken from representatives of the municipality for conducting the interview. All respondents were asked to sign a prior informed- consent form after the objectives and possible consequences of the study had been explained. The prior informed consent (PIC) form was translated into the local Pahari language.

\section{REFERENCES}

[1] Abbasi, A. M., Khan, M. A., Ahmad, M., Qureshi, R., Arshad, M., Jahan, S., Zafar, M., Sultana, S. (2010): Ethnobotanical study of wound healing herbs among the tribal communities in Northern Himalaya Ranges District Abbottabad, Pakistan. - Pakistan Journal of Botany 6: 3747-3753.

[2] Abbasi, A. M., Khan, M. A., Shah, M. H., Shah, M. M., Pervez, A., Ahmad, M. (2013a): Ethnobotanical appraisal and cultural values of medicinally important wild edible vegetables of Lesser Himalayas-Pakistan. - Journal of Ethnobiology and Ethnomedicine 9(1): 1.

[3] Abbasi, A. M., Khan, S. M., Ahmad, M., Khan, M. A., Quave, C. L., Pieroni, A. (2013b): Botanical ethnoveterinary therapies in three districts of the Lesser Himalayas of Pakistan. - Journal of Ethnobiology and Ethnomedicine 9.

[4] Abbasi, A. M., Shah, M. H., Li, T., Fu, X., Guo, X., Liu, R. H. (2015): Ethnomedicinal values, phenolic contents and antioxidant properties of wild culinary vegetables. Journal of Ethnopharmacology 162: 333-345.

[5] Abe, R., Ohtani, K. (2013): An ethnobotanical study of medicinal plants and traditional therapies on Batan Island, the Philippines. - Journal of Ethnopharmacology 145(2): 554565 .

[6] Adejuwon, A., Femi-Akinlosotu, O., Omirinde, J., Owolabi, O., Afodun, A. (2014): Launaea taraxacifolia ameliorates cisplatin-induced hepato-renal injury - European Journal of Medicinal Plants 4(5): 528.

[7] Afolayan, A. J., Grierson, D. S., Mbeng, W. O. (2014): Ethnobotanical survey of medicinal plants used in the management of skin disorders among the Xhosa communities of the Amathole District, Eastern Cape, South Africa. - Journal of ethnopharmacology 153(1): 220-232.

[8] Ahmad, K. S., Kayani, W. K., Hameed, M., Ahmad, F., Nawaz, T. (2012): Floristic diversity and ethnobotany of Senhsa, District Kotli, Azad Jammu \& Kashmir (Pakistan). - Pakistan Jouranl of Botany 44: 195-201.

[9] Ahmad, M., Sultana, S., Fazl-i-Hadi, S., ben Hadda, T., Rashid, S., Zafar, M., Khan, M. A., Khan, M. P. Z., Yaseen, G. (2014): An Ethnobotanical study of Medicinal Plants in high mountainous region of Chail valley (District Swat-Pakistan). - Journal of Ethnobiology and Ethnomedicine 10(36): 4269-10.

[10] Ahmed, E., Arshad, M., Saboor, A., Qureshi, R., Mustafa, G., Sadiq, S. and Chaudhari, S. K. (2013a): Ethnobotanical appraisal and medicinal use of plants in Patriata, New Murree, evidence from Pakistan. - Journal of Ethnobiology and Ethnomedicine 9(1): 13.

[11] Ahmed, E., Arshad, M., Saboor, A., Qureshi, R., Mustafa, G., Sadiq, S., Chaudhari, S. K. (2013b): Ethnobotanical appraisal and medicinal use of plants in Patriata, New Murree, evidence from Pakistan. - Journal of Ethnobiolology and Ethnomedicine 9: 13. 
[12] Ahmed, M., Khan, N., Wahab, M., Hamza, S., Siddiqui, M. F., Nazim, K., Khan, M. U. (2009): Vegetation structure of Olea ferruginea Royle forests of lower Dir District of Pakistan. - Pakistan Journal of Botany 41(6): 2683-2695.

[13] Ahmed, M., Trisha, U. K., Shaha, S. R., Dey, A. K., Rahmatullah, M. (2015): An initial report on the antihyperglycemic and antinociceptive potential of Lablab purpureus beans. - World Jounal of Pharmacy and Pharmaceutical Sciences 4(10): 95-105.

[14] Ajaib, M., Khan, Z.-u.-D., Khan, N., Wahab, M. (2010): Ethnobotanical studies on useful shrubs of district Kotli, Azad Jammu \& Kashmir, Pakistan. - Pakistan Journal of Botany 42(3): 1407-1415.

[15] Akhtar, N., Rashid, A., Murad, W. and Bergmeier, E. (2013): Diversity and use of ethnomedicinal plants in the region of Swat, North Pakistan. - Journal of Ethnobiology and Ethnomedicine 9(1):25.

[16] Al-Musayeib, N. M., Mothana, R. A., Matheeussen, A., Cos, P., Maes, L. (2012): In vitro antiplasmodial, antileishmanial and antitrypanosomal activities of selected medicinal plants used in the traditional Arabian Peninsular region. - BMC complementary and alternative medicine 12(1): 49.

[17] Ali, N., Shah, S. W. A., Shah, I., Ahmed, G., Ghias, M., Khan, I., Ali, W. (2012): Anthelmintic and relaxant activities of Verbascum Thapsus Mullein. - BMC complementary and alternative medicine 12(1): 29.

[18] Alonso-Castro, A. J., Maldonado-Miranda, J. J., Zarate-Martinez, A., del Rosario JacoboSalcedo, M., Fernández-Galicia, C., Figueroa-Zuñiga, L. A., Rios-Reyes, N. A., de LeónRubio, M. A., Medellín-Castillo, N. A., Reyes-Munguia, A. (2012): Medicinal plants used in the Huasteca Potosina, Mexico. - Journal of ethnopharmacology 143(1): 292-298.

[19] Amjad, M. S., Arshad, M. (2014): Ethnobotanical inventory and medicinal uses of some important woody plant species of Kotli, Azad Kashmir, Pakistan. - Asian Pacific Journal of Tropical Biomedicine 4(12): 952-958.

[20] Amjad, M. S., Arshad, M., Qureshi, R. (2015): Ethnobotanical inventory and folk uses of indigenous plants from Pir Nasoora National Park, Azad Jammu and Kashmir. - Asian Pacific Journal of Tropical Biomedicine 5(3): 234-241.

[21] Amjad, M. S., faisal Qaeem, M., Ahmad, I., Khan, S. U., Chaudhari, S. K., Malik, N. Z., Shaheen, H., Khan, A. M. (2017): Descriptive study of plant resources in the context of the ethnomedicinal relevance of indigenous flora: A case study from Toli Peer National Park, Azad Jammu and Kashmir, Pakistan. - PLoS One 12(2): e0171896.

[22] Arnold, B. C. (2015): Pareto distribution. - Wiley Online Library.

[23] Asad, M., Sabih, D., Chaudhory, B., Ahmad, I., Hussain, M., Izhar, N., Akmal, N., Shahzad, A.,Hussain, I. (2014): Anti-hemolytic property of local medicinal plant (s) upon Pakistani cobra venom induced hemolysis. - Journal of Animal and Plant Sciences 24:1701-1708.

[24] Asgarpanah, J., Kazemivash, N. (2013): Phytochemistry, pharmacology and medicinal properties of Carthamus tinctorius L. - Chinese Journal of Integrative Medicine 19(2): 153-159.

[25] Bano, A., Ahmad, M., Hadda, T. B., Saboor, A., Sultana, S., Zafar, M., Khan, M. P. Z., Arshad, M., Ashraf, M. A. (2014): Quantitative ethnomedicinal study of plants used in the skardu valley at high altitude of Karakoram-Himalayan range, Pakistan. - Journal of Ethnobiology and Ethnomedicine 10(1): 43.

[26] Barkatullah, B., Ibrar, M. (2011): Plants profile of Malakand Pass Hills, District Malakand, Pakistan. - African Journal of Biotechnology 10(73): 16521-16535.

[27] Basri, F., Sharma, H., Firdaus, S., Jain, P., Ranjan, A. (2014): A review of ethnomedicinal plant-Vitex negundo Linn. - International Journal 2(3): 882-894.

[28] Baydoun, S., Chalak, L., Dalleh, H., Arnold, N. (2015): Ethnopharmacological survey of medicinal plants used in traditional medicine by the communities of Mount Hermon, Lebanon. - Journal of Ethnopharmacology 173: 139-156. 
[29] Bharath, M., Tulasi, E. L. R., Sudhakar, K., Eswaraiah, M. C. (2013): Dalbergia sissoo dc.-an important medicinal plant. - Internatinal Journal of Research in Pharmacy and Chemistry 3(2): 385-8.

[30] Bhatia, H., Sharma, Y. P., Manhas, R., Kumar, K. (2015): 'Traditional phytoremedies for the treatment of menstrual disorders in district Udhampur, J\&K, India. - Journal of Ethnopharmacology 160: 202-210.

[31] Bibi, T., Ahmad, M., Tareen, R. B., Tareen, N. M., Jabeen, R., Rehman, S.-U., Sultana, S., Zafar, M., Yaseen, G. (2014): Ethnobotany of medicinal plants in district Mastung of Balochistan province-Pakistan. - Journal of Ethnopharmacology 157: 79-89.

[32] Borborah, K., Dutta, B., Borthakur, S. (2014): Traditional Uses of Allium L. Species from North East India with Special Reference to their Pharmacological Activities. American Journal of Phytomedicine and Clinical Therapeutics 2(8): 1037-1051.

[33] Borgatti, S. P. (1996): ANTHROPAC 4.0', Natick, MA: Analytic Technologies.

[34] Bruschi, P., Morganti, M., Mancini, M., Signorini, M. A. (2011): Traditional healers and laypeople: a qualitative and quantitative approach to local knowledge on medicinal plants in Muda (Mozambique). - Journal of ethnopharmacology 138(2): 543-563.

[35] Carrió, E.,Vallès, J. (2012): Ethnobotany of medicinal plants used in Eastern Mallorca (Balearic Islands, Mediterranean Sea). - Journal of Ethnopharmacology 141(3): 10211040 .

[36] Chidambaram, A. Aruna, A. (2013): Pharmacognostic study and development of quality parameters of whole plants of Trichodesma indicum (Linn.) R. - Br. Asian J. Pharm. Clin. Res 6(3): 167-169.

[37] Cruz, C. D. (2013): Genes: a software package for analysis in experimental statistics and quantitative genetics. - Acta Scientiarum. Agronomy 35(3): 271-276.

[38] de Albuquerque, U. P., Soldati, G. T., Sieber, S. S., Ramos, M. A., de Sá, J. C., de Souza, L. C. (2011): The use of plants in the medical system of the Fulni-ô people (NE Brazil): a perspective on age and gender. - Journal of ethnopharmacology 133(2): 866-873.

[39] del Carmen Juárez-Vázquez, M., Carranza-Álvarez, C., Alonso-Castro, A. J., GonzálezAlcaraz, V. F., Bravo-Acevedo, E., Chamarro-Tinajero, F. J., Solano, E. (2013): Ethnobotany of medicinal plants used in Xalpatlahuac, Guerrero, Mexico. - Journal of Ethnopharmacology 148(2): 521-527.

[40] Gangwar, A. K., Ghosh, A. K. (2014): Medicinal uses and Pharmacological activity of Adhatoda vasica. - International Journal of Herbal Medicine 2(1): 88-91.

[41] Govaerts, R. (2001): How many species of seed plants are there? - Taxon 50(4): 10851090.

[42] Goyal, A., Sharma, V., Upadhyay, N., Gill, S., Sihag, M. (2014). Flax and flaxseed oil: an ancient medicine \& modern functional food. - Journal of Food Science and Technology 51(9): 1633-1653.

[43] Heinrich, M., Ankli, A., Frei, B., Weimann, C., Sticher, O. (1998). Medicinal plants in Mexico: Healers' consensus and cultural importance. - Social Science \& Medicine, 47(11): 1859-1871.

[44] Hussain, A. I., Rathore, H. A., Sattar, M. Z., Chatha, S. A., Sarker, S. D., Gilani, A. H. (2014): Citrullus colocynthis (L.) Schrad (bitter apple fruit): A review of its phytochemistry, pharmacology, traditional uses and nutritional potential. - Journal of Ethnopharmacology 155(1): 54-66.

[45] Iqbal, H., Sher, Z., Khan, Z. U. (2011): Medicinal plants from salt range Pind Dadan Khan, district Jhelum, Punjab, Pakistan. - Journal of Medicinal Plants Research 5(11): 2157-2168.

[46] Ishtiaq, M., Mahmood, A., Maqbool, M. (2015): Indigenous knowledge of medicinal plants from Sudhanoti district (AJK), Pakistan. -Journal of Ethnopharmacology 168: 201207. 
[47] Ishtiaq, M., Maqbool, M., Hussain, T., Shah, A. (2013): Role of indigenous knowledge in biodiversity conservation of an area: A case study on tree ethnobotany of Soona Valley, District Bhimber Azad Kashmir, Pakistan. - Pakistan Journal of Botany 45: 157-164.

[48] Jarić, S., Popović, Z., Mačukanović-Jocić, M., Djurdjević, L., Mijatović, M., Karadžić, B., Mitrović, M., Pavlović, P. (2007): An ethnobotanical study on the usage of wild medicinal herbs from Kopaonik Mountain (Central Serbia). - Journal of Ethnopharmacology 111(1): 160-175.

[49] Jemâa, J. M. B., Haouel, S., Bouaziz, M., Khouja, M. L. (2012): Seasonal variations in chemical composition and fumigant activity of five Eucalyptus essential oils against three moth pests of stored dates in Tunisia. - Journal of Stored Products Research 48: 61-67.

[50] Joshi, Y., Joshi, A. K., Prasad, N., Juyal, D. (2014): A review on Ficus palmata (Wild Himalayan Fig). - Journal of Phytopharmacology 3(5):374-377.

[51] Kadam, P., Bhalerao, S. (2010): Sample size calculation. - International Journal of Ayurveda Research 1(1): 55.

[52] Kaleem, W. A., Muhammad, N., Khan, H., Rauf, A. (2014): Pharmacological and phytochemical studies of Genus Zizyphus. - Middle-East Journal of Science and Research 21: 1243-1263.

[53] Khan, I., AbdElsalam, N. M., Fouad, H., Tariq, A., Ullah, R., Adnan, M. (2014): Application of ethnobotanical indices on the use of traditional medicines against common diseases. - Evidence-Based Complementary and Alternative Medicine.

[54] Khan, M., Khan, M. A., Mujtaba, G., Hussain, M. (2012): Ethnobotanical study about medicinal plants of Poonch valley Azad Kashmir. - Journal of Animal Plant Science 22: 493-500.

[55] Khan, M. A., Khan, M. A., Hussain, M., Ghulam, G. M. (2010): An ethnobotanical inventory of Himalayan region Poonch valley Azad Kashmir (Pakistan). - Ethnobotany Research and Applications 8: 107-123.

[56] Khan, M. I., Khan, M. R. (2013): Gastroprotective potential of Dalbergia sissoo roxb. stem bark against diclofenac-induced gastric damage in rats. - Osong public health and research perspectives 4(5): 271-277.

[57] Khiljee, S., Rehman, N. U., Khiljee, T., Ahmad, R. S., Khan, M. Y., Qureshi, U. A. (2016): Use of traditional herbal medicines in the treatment of eczema. - Journal of Pakistan Association of Dermatology 21(2): 112-117.

[58] Locher, F. W. (2013): Cement: principles of production and use. - Verlag Bau+ Technik.

[59] Logan, M. H. (1986): Informant consensus: a new approach for identifying potentially effective medicinal plants. - Plants in indigenous medicine and diet: Biobehavioral approaches 91 .

[60] Lulekal, E., Asfaw, Z., Kelbessa, E., Van Damme, P. (2013): Ethnomedicinal study of plants used for human ailments in Ankober District, North Shewa Zone, Amhara region, Ethiopia. - Journal of Ethnobiology and Ethnomedicine 9(63): 4269-9.

[61] Mahmood, A., Mahmood, A., Mujtaba, G., Mumtaz, M. S., Kayani, W. K., Khan, M. A. (2012): Indigenous medicinal knowledge of common plants from district Kotli Azad Jammu and Kashmir Pakistan. -Journal of Medicinal Plant and Research 6: 4961-4967.

[62] Mahmood, A., Mahmood, A., Shaheen, H., Qureshi, R. A., Sangi, Y., Gilani, S. A. (2011a): Ethno medicinal survey of plants from district Bhimber Azad Jammu and Kashmir, Pakistan. - Journal of Medicinal Plants Research 5(11): 2348-2360.

[63] Mahmood, A., Riffat, N., Zabta, K., Aqeel, M. (2011b): Ethnobotanical survey of plants from Neelum, Azad Jammu and Kashmir, Pakistan. - Pakistan Journal of Botany 43: 105110.

[64] Mahmood, A., Riffat, N., Zabta, K., Aqeel, M. (2011c): Ethnobotanical survey of plants from Neelum, Azad Jammu and Kashmir, Pakistan. - Pakistan Journal of Botany 43(105): 10.

[65] Mahwasane, S., Middleton, L., Boaduo, N. (2013): An ethnobotanical survey of indigenous knowledge on medicinal plants used by the traditional healers of the 
Lwamondo area, Limpopo Province, South Africa. - South African Journal of Botany 88: 69-75.

[66] Mesfin, K., Tekle, G., Tesfay, T. (2013): Ethnobotanical study of traditional medicinal plants used by indigenous people of Gemad District, Northern Ethiopia. - Journal of Medicinal Plants Studies 1(4).

[67] Mouterde, P. (1966): Vicia in Nouvelle Flore du Liban et de la Syrie. - Librairie Orientale, Beirut 2 (396): 14.

[68] Mouterde, P. (1978): Nouvelle flore du Liban et de la Syrie: tome 3. Texte: premiere livraison. - Beyrouth: Dar El Machreq Editeurs 112p. Keys. Geog, 2.

[69] Mouterde, P. (1983): Nouvelle flore du Liban et de la Syrie: tome 3. Texte: quatrieme livraison. - Beyrouth, Dar El-Machreq SARL, 365-578.

[70] Nedelcheva, A., Dogan, Y. (2015): An ethnobotanical study on wild medicinal plants sold in the local markets at both sides of the Bulgarian-Turkish border. - Planta medica 81(16): PW_13.

[71] Njoroge, G. N., Bussmann, R. W. (2007): Ethnotherapeautic management of skin diseases among the Kikuyus of Central Kenya. - Journal of Ethnopharmacology 111(2): 303-307.

[72] Oyelere, E., Balogun, J., Abubakar, B. (2016): Growth and nutrient utilization of African catfish (Clarias gariepinus burchell) fed varying levels of Albizia lebbeck (benth) leaf meal. - Agrosearch 16(1): 13-22.

[73] Panyaphu, K., Van On, T., Sirisa-ard, P., Srisa-nga, P., ChansaKaow, S., Nathakarnkitkul, S. (2011): Medicinal plants of the Mien (Yao) in Northern Thailand and their potential value in the primary healthcare of postpartum women. - Journal of Ethnopharmacology 135(2): 226-237.

[74] Puri, R. K. (2010): Documenting local environmental knowledge and change. - In Conducting Research in Conservation, Routledge 168-191.

[75] Quinlan, M. (2005): Considerations for collecting freelists in the field: examples from ethobotany. - Field methods 17(3): 219-234.

[76] Qureshi, R., Bhatti, G. R., Memon, R. A. (2010): Ethnomedicinal uses of herbs from northern part of Nara desert, Pakistan. - Pakistan Journal of Botany 42(2): 839-51.

[77] Qureshi, R., Waheed, A., Arshad, M., Umbreen, T. (2009): Medico-ethnobotanical inventory of tehsil Chakwal, Pakistan. - Pakistan Journal of Botany 41(2): 529-538.

[78] Rana, P. K., Kumar, P., Singhal, V. K., Rana, J. C. (2014): Uses of local plant biodiversity among the tribal communities of Pangi Valley of district Chamba in cold desert Himalaya, India. - The Scientific World Journal.

[79] Rehman, N.-u., Khan, A.-u., Alkharfy, K. M., Gilani, A.-H. (2012): Pharmacological basis for the medicinal use of Lepidium sativum in airways disorders. - Evidence-Based Complementary and Alternative Medicine.

[80] Saluja, P., Shrivastava, K. (2011): Medicinal importance of weeds found in urban area of Raipur (CG). - Indian J. Applied \& Pure Bio 26(1): 159-170.

[81] Saqib, Z., Mahmood, A., Malik, R. N., Mahmood, A., Syed, J. H., Ahmad, T. (2014): Indigenous knowledge of medicinal plants in Kotli Sattian, Rawalpindi district, Pakistan. - Journal of Ethnopharmacology 151(2): 820-828.

[82] Schippmann, U., Leaman, D. J., Cunningham, A. (2002): Impact of cultivation and gathering of medicinal plants on biodiversity: global trends and issues. - In: Biodiversity and the ecosystem approach in agriculture, forestry and fisheries. FAO, pp.142-167.

[83] Shaheen, H., Qaseem, M. F., Amjad, M. S., Bruschi, P. (2017a): Exploration of ethnomedicinal knowledge among rural communities of Pearl Valley; Rawalakot, District Poonch Azad Jammu and Kashmir. - PLoS One 12(9): e0183956.

[84] Shaheen, H., Qureshi, R., Akram, A., Gulfraz, M., Potter, D. (2014a): A preliminary floristic checklist of Thal Desert Punjab, Pakistan. - Pakistan Jouranl of Botany 46(1): $13-18$. 
[85] Shaheen, H., Qureshi, R., Iqbal, S., Qasem, M. F. (2014b): Seasonal availability and palatability of native flora of Santh Saroola Kotli Sattian, Rawalpindi, Pakistan. - African Journal of Plant Science 8(2): 92-102.

[86] Shaheen, H., Qureshi, R., Qaseem, M. F., Amjad, M. S., Bruschi, P. (2017b): The cultural importance of indices: A comparative analysis based on the useful wild plants of Noorpur Thal Punjab, Pakistan. - European Journal of Integrative Medicine 12: 27-34.

[87] Shaukat, S. A., Shah, S. Z. A., Ahmad, M. J., Shaukat, S. K., Shoukat, S. W. (2012): Ethnobotanical study of some medicinal plants of union council Bangoin, Tehsil Rawalakot, AJ\&K. - Agricultural Advances 1(4): 105-109.

[88] Smith, J. J. (1993): Using ANTHOPAC 3.5 and a spreadsheet to compute a free-list salience index. - CAM 5(3): 1-3.

[89] Srivastava, R., Saluja, D., Dwarakanath, B. S., Chopra, M. (2011): Inhibition of human cervical cancer cell growth by ethanolic extract of Boerhaavia diffusa Linn.(punarnava) root. - Evidence-Based Complementary and Alternative Medicine.

[90] Torres-Avilez, W., Medeiros, P. M. D., Albuquerque, U. P. (2016): Effect of Gender on the Knowledge of Medicinal Plants: Systematic Review and Meta-Analysis. - EvidenceBased Complementary and Alternative Medicine.

[91] Ullah, M., Khan, M. U., Mahmood, A., Malik, R. N., Hussain, M., Wazir, S. M., Daud, M., Shinwari, Z. K. (2013): An ethnobotanical survey of indigenous medicinal plants in Wana district south Waziristan agency, Pakistan. - Journal of Ethnopharmacology 150(3): 918-924.

[92] Uniyal, B., Shiva, V. (2005): Traditional knowledge on medicinal plants among rural women of the Garhwal Himalaya, Uttaranchal. - Indian Journal of Traditional Knowledge 4(3):259-266.

[93] Vijayakumar, S., Yabesh, J. M., Prabhu, S., Manikandan, R., Muralidharan, B. (2015): Quantitative ethnomedicinal study of plants used in the Nelliyampathy hills of Kerala, India. - Journal of Ethnopharmacology 161: 238-254.

[94] Vijaykumar, V., Vanathi, P., Kanagasapabathy, P. (2015): Modified adaptive filtering algorithm for noise cancellation in speech signals. - Elektronika ir elektrotechnika 74(2): 17-20.

[95] Weller, S. C., Baer, R. (2002): Measuring within-and between-group agreement: Identifying the proportion of shared and unique beliefs across samples. - Field Methods 14(1): 6-25.

[96] Yaseen, G., Ahmad, M., Sultana, S., Alharrasi, A. S., Hussain, J., Zafar, M. (2015): Ethnobotany of medicinal plants in the Thar Desert (Sindh) of Pakistan. - Journal of Ethnopharmacology 163: 43-59.

[97] Zanoli, P., Rivasi, M., Zavatti, M., Brusiani, F., Vezzalini, F., Baraldi, M. (2005): Activity of single components of Ferula hermonis on male rat sexual behavior. International Journal of Impotence Research 17(6): 513-518. 